\title{
Messung der Qualität in Dienstleistungscentern - am Beispiel eines Flughafens
}
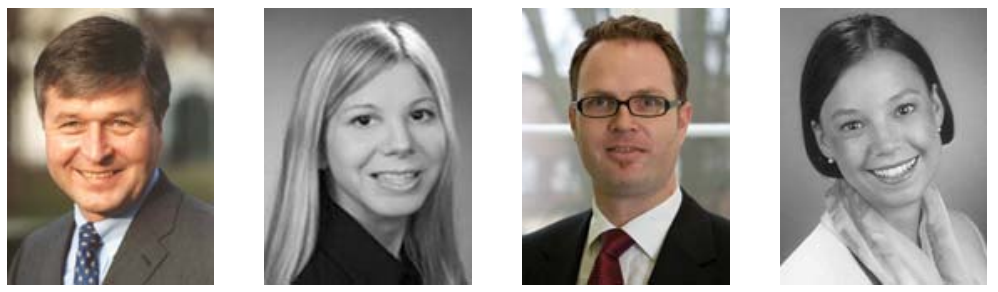

Manfred Bruhn • Verena Batt • Karsten Hadwich • Sandrina Meldau

Zusammenfassung: Dienstleistungsanbieter bieten ihre Leistungen vermehrt in Kooperation mit anderen Unternehmen in Form von Dienstleistungscentern an. Der Zusammenschluss von Unternehmen in Form eines Centers hat zur Folge, dass sich die Messung der Qualität deutlich komplexer gestaltet als bei einer einzelnen Organisation. Der vorliegende Artikel nimmt sich dieser Problematik an und verfolgt die Zielsetzung, ein theoriegeleitetes Modell zu entwickeln, das sich zur Messung der Qualität in Dienstleistungscentern eignet. Das Augenmerk bei dem zu konzeptualisierenden Modell liegt darauf, diejenigen Einflussfaktoren, die zur Gesamtqualitätswahrnehmung des Dienstleistungscenters beitragen, zu identifizieren. Es wird herausgearbeitet, dass sich die Qualitätsbeurteilung durch den Centernutzer zum einen auf Basis von Einzelinteraktionen (im Sinne

Eingegangen: 10.09 .2008 / Online publiziert: 03.02 .2010

(C) Gabler-Verlag 2010

Prof. Dr. M. Bruhn ( $\bowtie)$ · Dipl.-Rom. V. Batt

Lehrstuhl für Marketing und Unternehmensführung

Wirtschaftswissenschaftliche Fakultät

Universität Basel, Peter Merian-Weg 6

4002 Basel, Schweiz

E-Mail: Manfred.Bruhn@unibas.ch

Dipl.-Rom. V. Batt

E-Mail: Verena.Batt@unibas.ch

Prof. Dr. K. Hadwich

Inhaber des Lehrstuhls für Dienstleistungsmanagement

Universität Hohenheim, Wollgrasweg 23

70593 Stuttgart, Deutschland

E-Mail: Karsten.Hadwich@uni-hohenheim.de

Dr. S. Meldau

Leiterin der Abteilung Service und Qualität im Bereich Privatkunden

Credit Suisse, Neugasse 18, 8810 Horgen, Schweiz

E-Mail: sandrina_meldau@web.de 
von Kontaktpunkten) mit verschiedenen Servicepartnern und zum anderen auf Basis bestehender institutioneller Rahmenbedingungen (d.h. dem Centerbetrieb) bildet. Das globale Qualitätsurteil bezüglich des Dienstleistungscenters eines Centernutzers ist folglich eine Funktion aus n Kontaktpunktqualitäten (mit k = 1 bis $n$ ) und der Centerbetriebsqualität. Am Beispiel eines Flughafens wird anschließend überprüft, inwiefern das entwickelte Modell empirischen Nachweis findet. Die empirische Überprüfung des Modells zeigt, dass die Centerbetriebsqualität sowie der erste und der letzte Kontaktpunkt, den ein Centernutzer durchläuft, einen zentralen Einfluss auf die Gesamtqualitätswahrnehmung des Kunden haben.

Schlüsselwörter: Dienstleistungscenterqualität · Kontaktpunktqualität · Centerbetriebsqualität

JEL Classification: M31

\section{Bedeutung der Qualitätsmessung in Dienstleistungscentern}

Die Dienstleistungsqualität erweist sich aufgrund der gestiegenen Kundenerwartungen sowie der sich verschärfenden Wettbewerbsbedingungen mehr denn je als ein zentraler Erfolgsfaktor. Aus diesem Grund nimmt die Messung und Steuerung der Dienstleistungsqualität sowohl in der Wissenschaft als auch in der Praxis einen hohen Stellenwert ein (Bauer et al. 2006, S. 1). ${ }^{1}$ Auffallend ist, dass sich die Forschungsarbeiten zumeist auf die Qualität einer in sich geschlossenen Dienstleistung (,Stand-alone-Dienstleistung“), die von einem einzelnen, mehr oder weniger hierarchisch geführten Dienstleistungsunternehmen erbracht wird, beziehen (Meffert u. Birkelbach 2000, S. 171); Benkenstein u. Zielke 2003, S. 407). Die Frage der Qualität in Dienstleistungscentern ist bisher jedoch weitestgehend vernachlässigt worden.

Eine der wenigen Ausnahmen stellt die Arbeit von Birkelbach (1993) dar. Diese befasst sich zwar mit der Qualität von zentral geführten, rechtlich unabhängigen Dienstleistungsanbietern, die ein verbundenes Angebot an einem gemeinsamen Standort erstellen - aufgrund der rein konzeptionellen Ausrichtung der Arbeit wird allerdings nicht detailliert auf Fragen der Qualitätsmessung eingegangen. Dies ist verwunderlich, denn in verschiedenen Dienstleistungsbranchen ist seit einigen Jahren ein Wandel hin zur Bildung so genannter Dienstleistungsnetzwerke zu beobachten (Benkenstein u. Zielke 2003, S. 407). Darunter ist zu verstehen, dass Dienstleistungen nicht mehr nur als Ergebnis einzelner Anbieter erstellt werden, sondern vielmehr das Ergebnis der Zusammenarbeit verschiedener Unternehmen sind. Eine besondere Form dieser Dienstleistungsnetzwerke sind die bereits erwähnten Dienstleistungscenter (Birkelbach 1993; Meffert u. Birkelbach 2000). Dabei handelt es sich um eine Agglomeration von mehreren, rechtlich selbständigen, jedoch wirtschaftlich voneinander unabhängigen Unternehmen (,Servicepartner“) unter einer einheitlichen Leitung eines Centermanagements an einem räumlich abgegrenzten Erstellungsort („Centerbetrieb“) (vgl. hierzu ausführlich Meffert u. Birkelbach 2000, S. 171 ff.). Typische Beispiele für Dienstleistungscenter sind Verkehrscenter wie Bahnhöfe oder Flughäfen sowie Messe- und Kongresscenter, Freizeitparks, Shopping Center und Kliniken.

Wie aus den Ausführungen hervorgeht, ist die Qualitätsmessung von Dienstleistungscentern im Rahmen der bisherigen Forschung unzureichend berücksichtigt. Das Ziel 
des vorliegenden Beitrages ist daher, auf Basis theoretischer Überlegungen ein Modell zur Messung der Qualität in Dienstleistungscentern zu entwickeln, um auf diese Weise die bestehende Forschungslücke zu schließen. Der Erkenntnisbeitrag liegt dabei insbesondere in der theoriegeleiteten Identifikation von Einflussfaktoren, die zur Gesamtqualitätswahrnehmung des Dienstleistungscenters beitragen.

Daraus ergibt sich folgende Kernfrage, die durch den vorliegenden Artikel beantwortet wird:

Wie kann die Qualität in einem Dienstleistungscenter erklärt und gemessen werden?

Zur Beantwortung der Kernfrage sind folgende Forschungsfragen zu beantworten:

(1) Welche Einflussfaktoren spielen bei der Bildung des Qualitätsurteils eine Rolle?

(2) Wie lässt sich die Qualität eines Dienstleistungscenters definieren?

(3) Wie kann die Entstehung der Dienstleistungscenterqualität in einem Modell abgebildet werden?

Das nachfolgende Kapitel widmet sich den Besonderheiten der Qualitätsmessung in Dienstleistungscentern. Hierbei wird auf die Herausforderungen der Qualitätsmessung, mit denen das Management von Dienstleistungscentern konfrontiert wird, eingegangen. Im Zentrum des dritten Kapitels steht zunächst die Entstehung der Qualität in Dienstleistungscentern. Hierbei gilt es, jene Einflussfaktoren, die zur Bildung des Gesamtqualitätsurteils eines Dienstleistungscenters beitragen, zu identifizieren (Beantwortung von Forschungsfrage 1). Dies dient als Grundlage für die sich anschließende Definition des Begriffs Dienstleistungscenterqualität (Beantwortung von Forschungsfrage 2). Im vierten Kapitel wird ein Kausalmodell zur Messung der Dienstleistungscenterqualität konzeptualisiert. Hierbei werden zum einen die Modellvariablen vorgestellt, zum anderen werden Forschungshypothesen zu den postulierten Zusammenhängen zwischen den Modellvariablen formuliert (Beantwortung von Forschungsfrage 3). Am Beispiel eines Flughafens wird das entwickelte Modell anschließend einer empirischen Überprüfung unterzogen. Hierbei liegt das Augenmerk zunächst auf der Operationalisierung der Konstrukte, bevor im Anschluss daran auf die Messung des Kausalmodells und die Interpretation der Ergebnisse eingegangen wird. Für einen Flughafen als Untersuchungsgegenstand wurde sich deshalb entschieden, weil dieser Dienstleistungscentertyp durch eine ausgeprägte Heterogenität der beteiligten Unternehmen gekennzeichnet ist und sich dadurch ein besonderer Erkenntnisgewinn im Hinblick auf den Beitrag einzelner Unternehmen zur Gesamtqualität erwarten lässt (vgl. hierzu auch Birkelbach 1993, S. 22 f.). Das sechste Kapitel bildet das Fazit mit Handlungsempfehlungen für das Qualitätsmanagement von Dienstleistungscentern.

\section{Besonderheiten der Qualitätsmessung in Dienstleistungscentern}

Im Vergleich zu einem einzelnen Anbieter sind bei der Qualitätsmessung eines Dienstleistungscenters - bestehend aus mehreren Servicepartnern - einige Besonderheiten zu beachten. Die als Dienstleistungscenter organisierten Anbieter offerieren dem Kunden ein Leistungsbündel, das aus einer Vielzahl individueller Leistungsversprechen besteht (Woratschek et al. 2003, S. 255). Die Nachfrager können aus dem Leistungsangebot auswählen, 
wobei sie während der Leistungsinanspruchnahme mit verschiedenen Unternehmen des Dienstleistungscenters in Kontakt treten (Bigné et al. 2003, S. 243 f.). Durch die Nutzung des Leistungsangebots entsteht eine Dienstleistungskette bzw. -sequenz, d. h., der Kunde nimmt die verschiedenen Unternehmen und deren Angebot isoliert wahr und reiht die einzelnen Ereignisse zu einem „,gesamthaften“ Dienstleistungserlebnis aneinander (Svensson 2004). Erst das Zusammenspiel der unterschiedlichen, einander ergänzenden Leistungen verschiedener Anbieter stiftet in ihrer Verbindung dem Nachfrager einen Nutzen (Wöhler 2001, S. 191). Der Kunde beurteilt die jeweiligen Interaktionen mit dem von ihm in Anspruch genommenen Servicepartnern sowie die in diesem Kontext erbrachte Leistung anhand seiner spezifischen Erwartungen. Die Beurteilung der Gesamtqualität des Dienstleistungscenters durch den Kunden setzt sich schließlich aus der wahrgenommen Qualität der Einzelerlebnissen zusammen (Spintig 2003, S. 2).

Es wird deutlich, dass der Zusammenschluss in Form eines Centers dazu führt, dass sich die Messung und Steuerung der Qualität deutlich komplexer gestaltet als bei einer einzelnen Organisation, da der Nachfrager mit verschiedenen Teilaspekten des Netzwerkangebotes in Kontakt kommt, bezüglich derer er sich ein Qualitätsurteil bilden kann (Birkelbach 1993, S. 3). Da es jedoch an Untersuchungen zur Qualitätsmessung in Dienstleistungscentern mangelt, ist auf Beiträge aus angrenzenden Forschungsbereichen zurückzugreifen, um Anhaltspunkte zur Qualitätsmessung in Dienstleistungscentern erhalten zu können. Ausgehend von den vorherigen Überlegungen, dass die in einem Dienstleistungscenter organisierten Anbieter dem Kunden (a) ein Leistungsbündel (mehrere Leistungen) offerieren und durch die Nutzung dieses Angebots (b) eine Dienstleistungskette (bestehend aus mehreren Kontaktpunkten) entsteht, bietet es sich an, von der Anzahl der Anbieter zu abstrahieren und sich auf Arbeiten zu konzentrieren, die sich zum einen mit der Qualität von Leistungsbündeln und zum anderen mit Dienstleistungsketten befassen. Bei einer Vielzahl von Arbeiten zu diesen Forschungsbereichen konnte nachgewiesen werden, dass sich bei Dienstleistungsbündeln die Gesamtqualität aus verschiedenen Teilleistungen zusammensetzt (z. B. bei Güthoff 1995; Olandt 1998; Bigné et al. 2003) bzw. bei Dienstleistungsketten die Gesamtqualität durch die einzelnen Teilphasen beeinflusst wird (z. B. bei Danaher u. Mattson 1994b; Riedmüller 2003).

Diese Erkenntnisse lassen sich auf den vorliegenden Kontext der Dienstleistungscenterqualität übertragen. So sind für die Messung der Gesamtqualität in Dienstleistungscentern die Einflussfaktoren der Gesamtqualität in Form von Teilqualitäten zu ermitteln. Hierfür kann es beispielsweise erforderlich sein, die gesamte Dienstleistungskette in einzelne Kontaktpunkte - hinter denen verschiedene Anbieter des Dienstleistungscenters stehen zu zerlegen sowie die Rahmenbedingungen, innerhalb derer sich der Dienstleistungsprozess abspielt, in die Betrachtung einzubeziehen. Eine isolierte Qualitätsmessung einzelner Teilqualitäten reicht jedoch nicht aus, denn sie beleuchtet immer nur einen Teilaspekt des gesamten Dienstleistungscenters. Daher gilt es zu analysieren, wie sich die einzelnen Teilqualitäten des Dienstleistungscenters bei der Bildung des Gesamtqualitätsurteils beeinflussen bzw. welche Abhängigkeiten zwischen den einzelnen Teilqualitäten bestehen (horizontale Interdependenzen). Des Weiteren ist zu untersuchen, in welchem Maße sich die Wahrnehmung von verschiedenen Teilqualitäten auf die Gesamtqualität des Dienstleistungscenters auswirkt (vertikale Interdependenzen). 


\section{Theoretische Überlegungen zur Erklärung der Qualität in Dienstleistungscentern}

Um die Servicequalität in einem Dienstleistungscenter zu analysieren ist es notwendig, ein grundlegendes Verständnis der Dienstleistungscenterqualität zu erhalten. In der Literatur besteht weitgehend Einigkeit darüber, dass sich die Gesamtqualität einer Dienstleistung aus verschiedenen Teilqualitäten zusammensetzt (z. B. Güthoff 1995, S. 80; Benkenstein u. Zielke 2003, S. 411 f.; Holzherr 2006). Um die Entstehung der Gesamtqualität erklären zu können, sind daher zunächst die inhaltlichen Teilqualitäten von Dienstleistungscentern zu identifizieren.

\subsection{Identifikation der Teilqualitäten}

Für die Identifikation der inhaltlichen Teilqualitäten wird im Folgenden auf den Ansatz von Donabedian (1980), der eine Unterscheidung der Dienstleistungsqualität in die drei Dimensionen Potenzial-, Prozess- und Ergebnisqualität vornimmt. ${ }^{2}$

Die Potenzialdimension umschreibt die Wahrnehmung der Strukturen und Potenziale des betrachteten Dienstleistungsanbieters. Allgemein können der Potenzialdimension alle mit dem Nachfrager in Kontakt kommenden Subjekte und Objekte eines Anbieters zugeordnet werden. Übertragen auf den vorliegenden Kontext lassen sich hierunter die Potenziale des Centerbetriebs, d. h. die centerbetriebsspezifischen Rahmenbedingungen, innerhalb derer sich der Dienstleistungsprozess abspielt (wie z. B. die Infrastruktur des Dienstleistungscenters, Leistungsumfang des Centerbetriebs) sowie die Potenziale der verschiedenen Servicepartner (z. B. Warenangebot, Geschäftseinrichtung), subsumieren.

Die Prozessdimension beschreibt die Wahrnehmung aller Aktivitäten während der Leistungserstellung. Damit wird der Tatsache Rechnung getragen, dass mit der Nutzung eines Dienstleistungscenters ein besonderes Integrationserfordernis des externen Faktors verbunden ist. Bezogen auf den Untersuchungsgegenstand Dienstleistungscenter handelt es sich hierbei um die Interaktion des Centernutzers mit den jeweiligen Servicepartnern des Centers.

Bei der Ergebnisdimension steht die Beurteilung des Leistungsergebnisses im Vordergrund. Die Ergebnisqualität nach Donabedian ist im vorliegenden Kontext als zusammenfassende Bewertung durch den Centernutzer zu verstehen und ist - wie die nachfolgenden Ausführungen zeigen - mit der Dienstleistungscenterqualität gleichzusetzen. Im Folgenden wird daher die Ergebnisqualität als eigenständige Teilqualität vernachlässigt.

Auf Basis dieser Überlegungen ist es möglich, strukturiert relevante Teilqualitäten eines Dienstleistungscenters zu identifizieren. Hierbei handelt es sich im Wesentlichen um die Potenziale des Centerbetriebs sowie die Wahrnehmung aller Aktivitäten während der Leistungserstellung durch die verschiedenen Servicepartner (vgl. Abb. 1).

\subsection{Entstehung der Gesamtqualität}

Nachdem die inhaltlichen Teilqualitäten identifiziert wurden, ist die Ermittlung der Gesamtqualität in einem Dienstleistungscenter zu definieren (vgl. hierzu Abb. 2). 


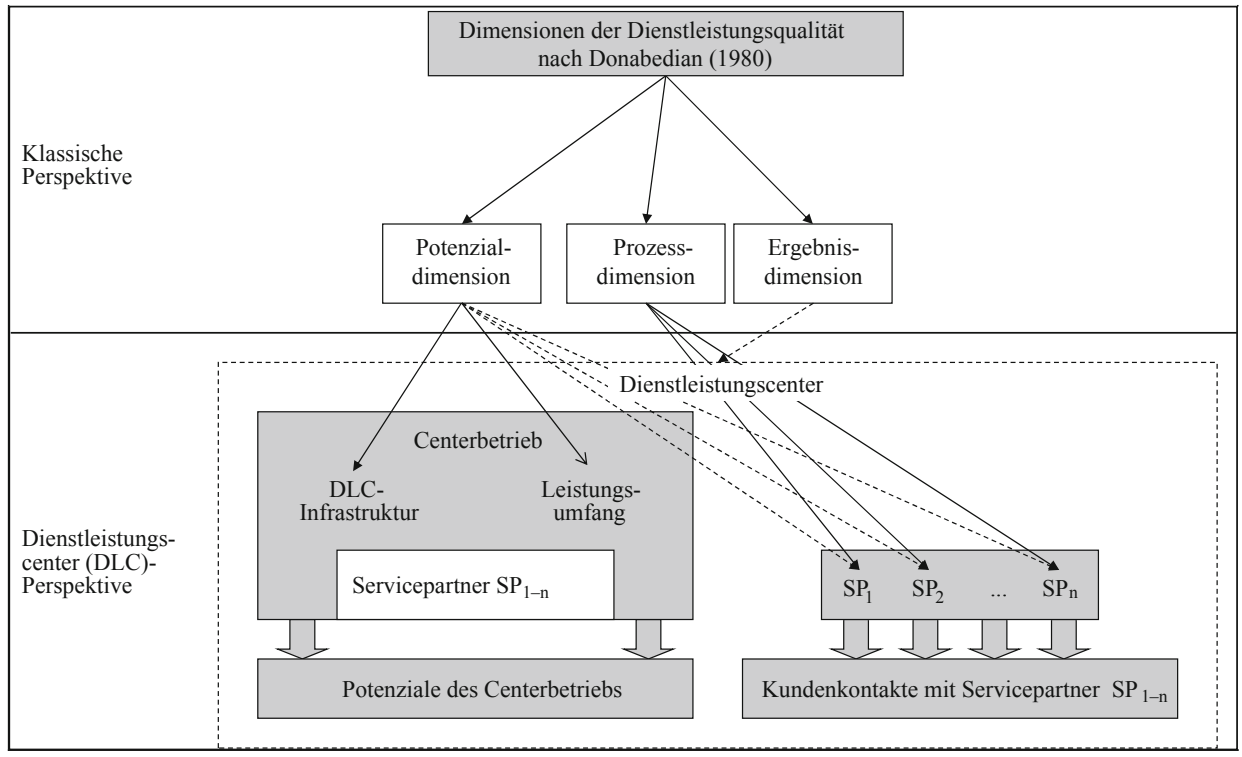

Abb. 1: Ableitung von Teilqualitäten in einem Dienstleistungscenter

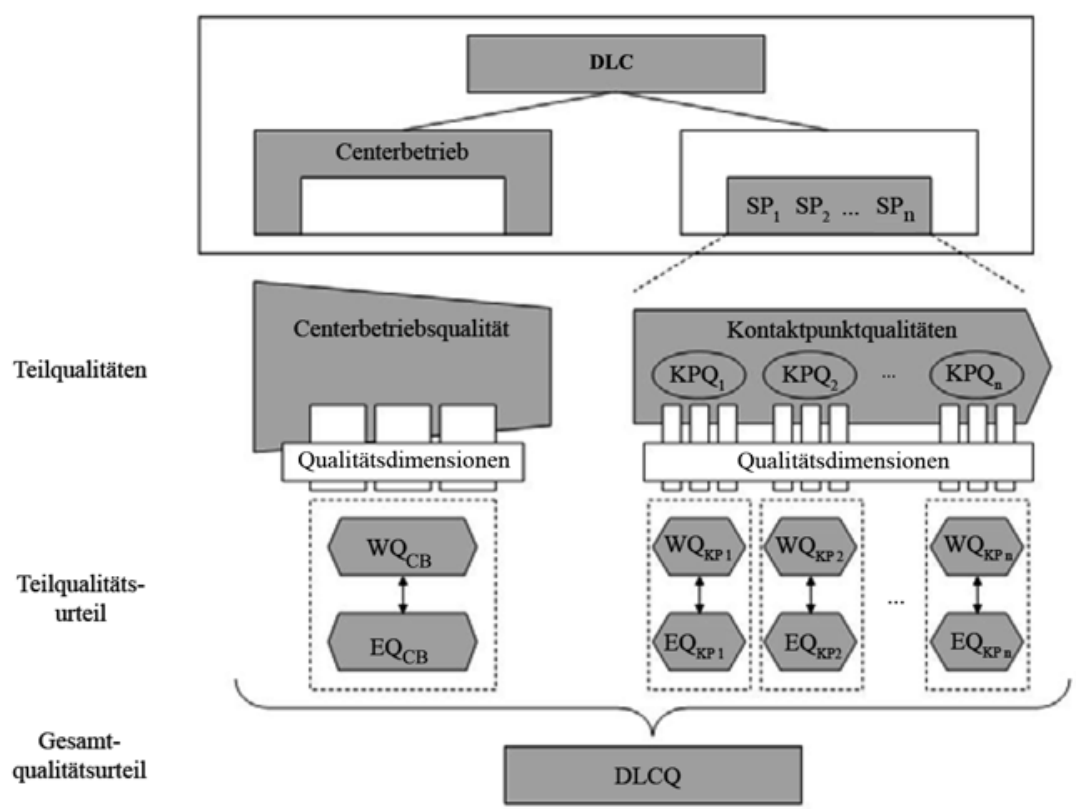

Abb. 2: Entstehung der Gesamtqualität in einem Dienstleistungscenter 
Um zu einem Teilqualitätsurteil zu kommen, beurteilt der Centernutzer die wahrgenommene Centerbetriebsqualität (WQ ${ }_{\mathrm{CB}}$ ) (Bewertung des Centerbetriebs durch den Centernutzer) sowie die wahrgenommene Qualität der in Anspruch genommenen Kontaktpunkte $\left(\mathrm{WQ}_{\mathrm{KP}}\right)$ (Bewertung des Kontaktpunktes durch den Centernutzer) des Dienstleistungscenters (vgl. ähnlich bei Riedmüller 2003, S. 111). Dabei zieht er zur Beurteilung centerbetriebsspezifische Merkmale bzw. kontaktpunktspezifische Merkmale heran. Entsprechend der vorangegangenen Ausführungen beziehen sich centerbetriebsspezifische Qualitätsmerkmale auf die institutionellen Rahmenfaktoren eines Centerbetriebs. Kontaktpunktspezifische Qualitätsmerkmale nehmen Bezug auf die Potenziale des Servicepartners sowie Einzelinteraktionen mit dem Servicepartner $\mathrm{SP}_{1-n}$. Die centerbetriebsspezifischen bzw. kontaktpunktspezifischen Merkmale können auf einem höheren Aggregationsniveau zu Qualitätsdimensionen zusammengefasst werden (Hentschel 2000, S. 295). Dem Bewertungsprozess liegt schließlich ein Abgleich zwischen der Anspruchs- und Eindruckskomponente (Soll-Ist-Vergleich) einer Qualitätsdimension bzw. der sie determinierenden Merkmale zugrunde (die Gleichsetzung der Teilqualitäten mit dem Grad der Erfüllung der Kundenerwartungen zielt auf GAP 5 des GAP-Modells ab, vgl. hierzu ausführlich Parasuraman et al. 1985). Die Anspruchskomponente kann als erwartete Qualität (EQ) bezeichnet werden und stellt somit die Soll-Größe dar, anhand derer die wahrgenommene Qualität (Ist-Größe) eingestuft wird. Das Anspruchsniveau der erwarteten Qualität hängt dabei stark von den Erfahrungen ab, die der Kunde im Rahmen von früheren Transaktionen mit dem entsprechenden Anbieter gemacht hat, aber auch aus ehemals in Anspruch genommenen Leistungsangeboten von direkten Konkurrenten oder vergleichbaren Leistungsanbietern. Das Urteil in Bezug auf die betrachtete Teilqualität fällt umso positiver aus, je mehr die Eindruckskomponenten der Qualitätsmerkmale (Ist-Größen) den Anspruchskomponenten (Soll-Größen) über alle Qualitätsdimensionen hinweg entsprechen. Von einer hohen Gesamtqualität eines Dienstleistungscenters ist schließlich zu sprechen, wenn der Centernutzer nach der Inanspruchnahme des Dienstleistungscenters alle Teilqualitäten, denen er eine besondere Bedeutung zuspricht, als positiv bewertet (vgl. ähnlich Parasuraman et al. 1985). Zusammenfassend kann folgende Begriffsdefinition der Dienstleistungscenterqualität abgeleitet werden:

Dienstleistungscenterqualität (DLCQ) ist die durch den Centernutzer anhand einzelner Qualitätsattribute wahrgenommene und hinsichtlich seiner Erwartungen bewertete Beschaffenheit aller centerbetriebspezifischen Rahmenfaktoren (Centerbetriebsqualität) sowie der gesamten Kundenkontaktpunkte (Kontaktpunktqualitäten) der Dienstleistungssequenz. Sie spiegelt sich im Grad der Erfüllung der Kundenanforderungen des Centernutzers wider.

\section{Konzeptualisierung eines Kausalmodells zur Dienstleistungscenterqualität}

Auf Basis der vorherigen Überlegungen wird ein Kausalmodell der Dienstleistungscenterqualität konzipiert, das in Abb. 3 dargestellt ist. Im Folgenden wird auf die einzelnen Konstrukte sowie auf die postulierten Zusammenhänge zwischen den jeweiligen Konstrukten eingegangen. 


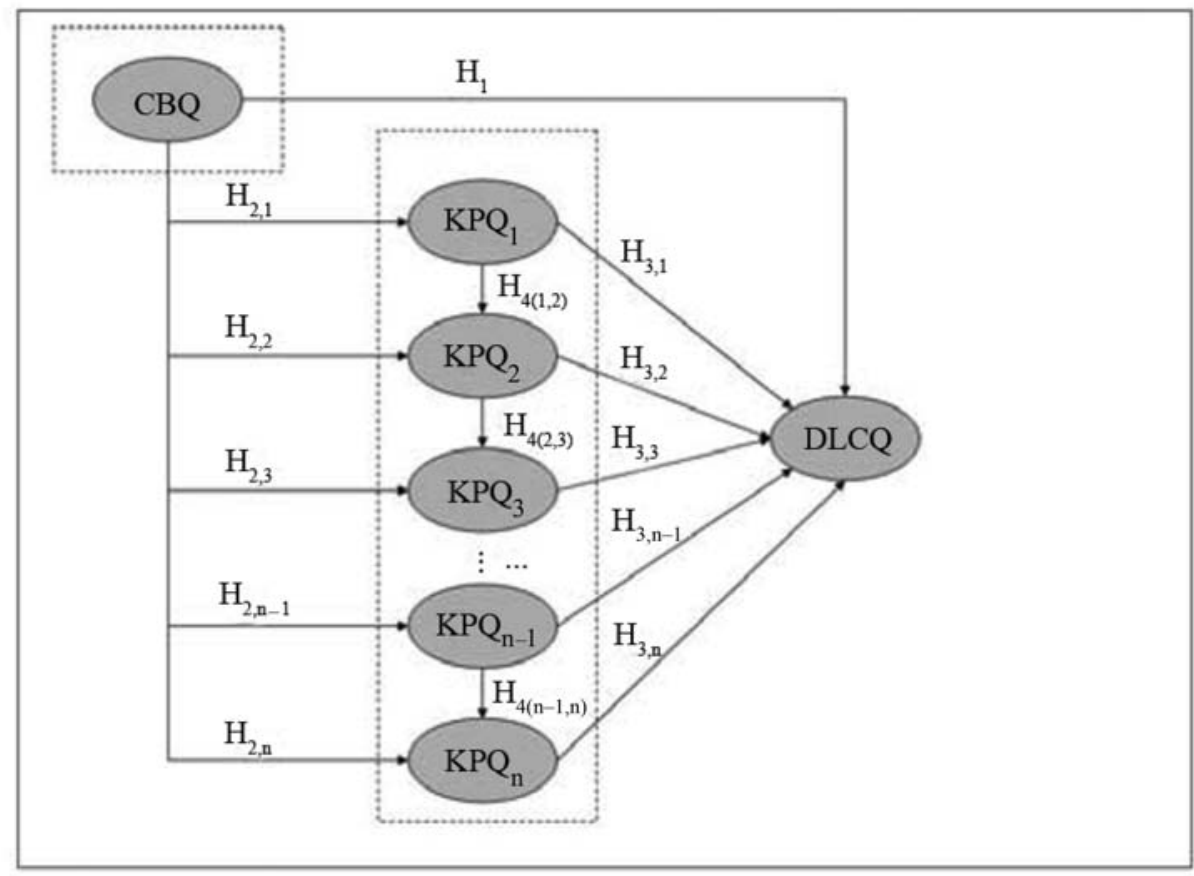

Abb. 3: Qualitätsmodell für Dienstleistungscenter

\subsection{Konzeptualisierung der Centerbetriebsqualität}

Der Centerbetrieb bildet den institutionellen Rahmen für die innerhalb des Dienstleistungscenters angesiedelten Servicepartner. Er untersteht der Leitung des Centermanagements mit eigenem Personal, den Gebäude- und Flächeneinheiten sowie den zugehörigen Ausstattungs- und Einrichtungselementen. Im Hinblick auf die Erfassung der Centerbetriebsqualität sind diejenigen Kontaktobjekte bzw. -subjekte ${ }^{3}$ des Centerbetriebs zu identifizieren, die vom Centernutzer subjektiv wahrgenommen werden. Es sind dies der Standort, die Gebäude- und Flächeneinheiten, die dazugehörige technische Ausstattung, die Mitarbeitenden des Centerbetriebs sowie der Leistungsumfang des Centerbetriebs (Birkelbach 1993, S. 12, 34).

Die Qualitätswahrnehmung des Standorts wird bestimmt durch die Verkehrsanbindung einschließlich der Ausschilderung und des vor Ort bestehenden Parkplatzangebots. Hat der Kunde den Dienstleistungscenter erreicht, gewinnt er einen Eindruck von Gebäude, Fläche sowie der zugehörigen technischen Ausstattung. Bei der Beurteilung von Gebäude- und Flächeneinheiten spielen sowohl materielle als auch nicht-materielle Faktoren eine Rolle (Bitner 1992, S. 60, 65 ff.; Birkelbach 1993, S. 43 ff.). Materielle Faktoren, die der Kunde während der Centernutzung wahrnimmt, stellen z. B. die Architektur, das Design sowie die Einrichtung des Centers dar. Zu den nicht direkt greifbaren Umfeldfaktoren, die die Qualitätswahrnehmung beeinflussen, zählen beispielsweise die Temperatur, die Luftqualität, der Geräuschpegel, das Sicherheitsempfinden, die Sauberkeit aber auch die Atmosphäre im 
Centerbetrieb (Becker 1981; Baker 1987; Baker et al. 1988; Bitner 1992). Die atmosphärische Qualität bezeichnet dabei - in Anlehnung an die Beschreibung der Ladenatmosphäre nach Bost (1987) - die „Stimmung, die der Centerbetrieb bei den Centernutzern auslöst““. Die technische Ausstattung wird in Bezug auf ihren technisch-optischen Erhaltungszustand und in ihrer Verfügbarkeit durch den Kunden evaluiert. Ferner können für die Qualitätswahrnehmung des Centerbetriebs die Centerbetriebsmitarbeitenden eine Rolle spielen. Ausschlaggebend hierfür ist der Anteil an Tätigkeiten mit direktem Kundenkontakt (FrontOffice-Tätigkeiten). Stellen Mitarbeitende des Centers für den Centernutzer Leistungen bereit, die der Kunde im Rahmen eines Interaktionsprozesses in Anspruch nehmen kann, zeigt sich die Personalqualität u. a. im kundengerichteten Verhalten und der Kompetenz der Centerbetriebsmitarbeitenden. Es ist jedoch davon auszugehen, dass der überwiegende Tätigkeitsblock sich auf Aufgaben bezieht, die unabhängig vom direkten Nachfragerkontakt sind (Back-Office-Tätigkeiten). Diese Tätigkeiten haben die Aufrechterhaltung bzw. Sicherstellung der Funktionsfähigkeit des Dienstleistungscenters zum Inhalt. Die Qualität dieser Tätigkeiten ist für den Centernutzer nur indirekt durch das Ergebnis der Arbeit erkennbar, d. h., das Gebäude ist sauber und gepflegt, die technischen Einrichtungen funktionieren, usw. Da sich diese Faktoren auf bereits genannte Aspekte (wie z. B. das Gebäude, die technische Ausstattung) der Centerbetriebsqualität beziehen, werden sie an dieser Stelle nicht als eigenständig bezüglich der Qualitätswahrnehmung durch den Centernutzer eingestuft und somit im Rahmen der Konzeptualisierung der Centerbetriebsqualität vernachlässigt. Schließlich wird die Wahrnehmung der Dienstleistungsqualität des Centerbetriebs durch den Leistungsumfang als eine Art „Querschnittsfunktion“ des Gesamtangebotes des Centerbetriebs geprägt. Von zentraler Bedeutung ist hierbei der gesamte „Servicepartnermix“. Die Anzahl der Servicepartner und die von ihnen erbrachten Funktionen spiegeln sich in der von den Centernutzern wahrgenommenen Angebotsbreite und -tiefe wider.

Die Gesamtqualität eines Dienstleistungscenters setzt sich aus der Centerbetriebsqualität und den Kontaktpunktqualitäten zusammen (vgl. Kap. 2). Hinsichtlich der Beziehung zwischen der Centerbetriebsqualität und der Dienstleistungscenterqualität kann demnach folgende Hypothese aufgestellt werden:

$H_{l}$ : Die Centerbetriebsqualität CBQ beeinflusst die Gesamtqualität des Dienstleistungscenters DLCQ direkt positiv.

Basierend auf den vorangegangenen Überlegungen wurden unter der Centerbetriebsqualität die Potenziale des Dienstleistungscenters zusammengefasst. Nach Donabedian (1980) stellt die Potenzialqualität eine Voraussetzung für die Prozess- und Ergebnisqualität dar. Aus diesem Grund kann davon ausgegangen werden, dass die Potenziale des Centerbetriebs (die Centerbetriebsqualität) auch den Leistungserstellungsprozess an den verschiedenen Kontaktpunkten im Rahmen der Dienstleistungssequenz beeinflussen. Entsprechend lässt sich folgende Hypothese bezüglich des Einflusses der Centerbetriebsqualität auf die Kontaktpunktqualitäten formulieren:

$\mathrm{H}_{2}$ : Die Centerbetriebsqualität CBQ beeinflusst die Kontaktpunktqualitäten $\mathrm{KPQ}_{\mathrm{k}}(\mathrm{k}=1$ bis $\mathrm{n}$ ) direkt positiv (n Teilhypothesen). 


\subsection{Konzeptualisierung der Kontaktpunktqualitäten}

Da der Centernutzer an jedem der Kontaktpunkte einen Eindruck von der Qualität der Leistungen eines Servicepartners gewinnt, beeinflussen - neben der Centerbetriebsqualität die Kontaktpunktqualitäten das Gesamtqualitätsurteil eines Dienstleistungscenters (vgl. Kap. 2; vgl. hierzu auch Albrecht 1988).

In der Wissenschaft gibt es bislang wenig Forschungsergebnisse zur „Kontaktpunktqualität“ bzw. „Service Encounter Quality“ (Jayawardhena et al. 2003, S. 2; Raajpoot 2004, S. 181 ff.; Jayawardhena et al. 2007, S. 575 ff.). Vor allem besteht keine Einigkeit über die inhaltlichen Facetten des Konstrukts (Brady u. Cronin 2001, S. 34). Aus diesem Grund erscheint eine Analyse der in der Literatur identifizierten Dimensionen der Dienstleistungsqualität hinsichtlich ihrer Bedeutung für einzelne Kontaktpunkte zweckmäßig.

Die inhaltsanalytische Betrachtung der einzelnen Qualitätsdimensionen zeigt, dass die Wahrnehmung der Qualität eines Dienstleistungsanbieters zu einem Großteil aus den Erfahrungen und Erkenntnissen, die der Nachfrager aus der Interaktion mit dem Kontaktpersonal im Rahmen des Erstellungsprozesses gewinnt, resultiert (z. B. Dabholkar et al. 1996; Brady u. Cronin 2001; Sureshchandar et al. 2003). Neben den Dimensionen, die direkt oder indirekt mit der Interaktion zwischen Anbieter und Kunden zusammenhängen, spielen die Leistung und die mit der Inanspruchnahme der Leistung zusammenhängenden Qualitätsdimensionen (umfeld-, nutzungs-, zeit- sowie sicherheitsbezogene Qualitätsdimensionen) für die Qualitätsbeurteilung eine zentrale Rolle (z. B. Bell et al. 1997; Owlia u. Aspinwall 1998; Siu u. Cheung 2001). Daher wird im Folgenden davon ausgegangen, dass sich die Beurteilung der Kontaktpunktqualitäten zum einen an der Interaktion zwischen dem Centernutzer und dem Servicepartnerkontaktpersonal und zum anderen an leistungsbezogenen Aspekten des jeweiligen Kontaktpunktes orientiert.

Die Ermittlung eines Gesamtqualitätsurteils des Dienstleistungscenters als einem dynamischen Wahrnehmungsobjekt, deren Teilqualitäten während der Nutzung durch ein zeitliches Neben- und Nacheinander gekennzeichnet sind, ist nach der Centernutzung mit einem statischen Wahrnehmungsobjekt vergleichbar. Entsprechend den Erkenntnissen der Wahrnehmungspsychologie werden komplexe statische Wahrnehmungsobjekte (z. B. ein Kunstwerk) zunächst hinsichtlich ihrer Bestandteile isoliert betrachtet und erst im Anschluss in ihrer Gesamtstruktur evaluiert (vgl. hierzu die Argumentation bei Güthoff 1995, S. $80 \mathrm{f}$. und zur Wahrnehmung komplexer Objekte Stadler et al. 1977). Übertragen auf Dienstleistungscenter kann daher angenommen werden, dass einzelne Teilbereiche auf einem gewissen Aggregationsniveau erst isoliert beurteilt und dann zu einem Gesamtqualitätsurteil integriert werden. Dies entspricht auch der vorangegangenen Argumentation, nach der die Gesamtqualität des Dienstleistungscenters durch die Centerbetriebsqualität und den verschiedenen Kontaktpunktqualitäten in Form von Teilqualitäten beeinflusst wird. Folglich wird nicht nur eine positive Kausalbeziehung zwischen der Centerbetriebsqualität und der Dienstleistungscenterqualität vermutet, sondern auch ein Wirkungszusammenhang zwischen den Kontaktpunktqualitäten und der Dienstleistungscenterqualität unterstellt.

$H_{3}$ : Die Kontaktpunktqualitäten $\mathrm{KPQ}_{\mathrm{k}}(\mathrm{k}=1$ bis $\mathrm{n})$ beeinflussen die Gesamtqualität des Dienstleistungscenters DLCQ direkt positiv (n Teilhypothesen). 
Die Centernutzung kann als ein Prozess beschrieben werden, in dem der Kunde mit einer Reihe von Servicepartnern an verschiedenen Kontaktpunkten in Kontakt kommt. Untersuchungen belegen, dass die Zufriedenheit eines Kunden am zweiten Kundenkontaktpunkt vom ersten Kontaktpunkt beeinflusst wird (Danaher u. Mattson 1994a, S. 7 und 1994b, S. 71; Bauer et al. 2000, S. 37). ${ }^{4}$ Für das Qualitätsurteil eines Centernutzers ist daher anzunehmen, dass zwischen der Qualitätswahrnehmung eines Kontaktpunktes und der Qualitätswahrnehmung des nachfolgenden Kontaktpunktes ein Zusammenhang besteht (vgl. zu ähnlichen Überlegungen Svensson 2006b, S. 56 f.).

Wird die Prämisse aufgehoben, dass sich diese Betrachtung auf Kontaktpunkte eines einzelnen Unternehmens bezieht, ist in Anlehnung an die Untersuchungsergebnisse eine kausale Abhängigkeit zwischen den Kontaktpunktqualitäten in der Dienstleistungssequenz einer Centernutzung zu vermuten, d. h., die wahrgenommene Qualität eines Kontaktpunktes beeinflusst die wahrgenommene Qualität des darauf folgenden Kontaktpunktes.

$H_{4}$ : Die Kontaktpunktqualität $\mathrm{KPQ}_{\mathrm{k}}$ beeinflusst die Kontaktpunktqualität $\mathrm{KPQ}_{\mathrm{k}+1}(\mathrm{k}=1$ bis $n$ ) direkt positiv (n-1 Teilhypothesen).

\section{Kausalanalytische Untersuchung zur Dienstleistungscenterqualität eines Flughafens}

\subsection{Identifizierung der Kontaktpunkte}

Im Zentrum der empirischen Untersuchung steht mit dem EuroAirport Basel-MulhouseFreiburg ein regionaler, mittelgroßer Verkehrsflughafen im Grenzgebiet Schweiz/Frankreich/Deutschland. Das Ziel ist es, die Gültigkeit des theoretisch hergeleiteten Qualitätsmodells zu überprüfen.

Zunächst ist der Prozess, den der Kunde bei Inanspruchnahme des Leistungsangebots eines Dienstleistungscenters durchläuft, in die aus Kundensicht relevanten Kundenkontaktpunkte zu zerlegen; mit dem Ziel, alle für die Qualitätswahrnehmung relevanten Kontaktpunkte zu identifizieren. Ausgangspunkt der Überlegungen ist hierbei, dass der Kunde während der Nutzung eines Dienstleistungscenters in einer gewissen Abfolge eine Reihe von Leistungen verschiedener Servicepartner in Anspruch nimmt. Zur Visualisierung des Serviceprozesses wurde auf Basis der Blueprinting-Methode ein Ablaufdiagramm erstellt (zur Blueprinting-Methode vgl. Shostack 2001). Hierfür wurden im Rahmen einer teilnehmenden, verdeckten Beobachtung zunächst verschiedene Kontaktpunkte und mögliche Passagierpfade eines abfliegenden Passagiers identifiziert. ${ }^{5}$ Zur Ergänzung der Ergebnisse aus der teilnehmenden Beobachtung dienten acht Experteninterviews ${ }^{6}$ und 16 persönliche Interviews mit Passagieren des Flughafens. ${ }^{7}$ Die Experten wurden gebeten, den typischen Kundenpfad, den ein Passagier nach seinem Eintreffen am Flughafen durchläuft, zu beschreiben. Die Passagiere wurden ebenfalls aufgefordert, die einzelnen Tätigkeiten zu benennen, die sie seit dem Eintreffen am Flughafen selber ausgeführt bzw. durchlaufen haben.

Auf Basis der so erhobenen qualitativen Daten erfolgte eine Visualisierung des Kundenprozesses für abfliegende Passagiere. Abb. 4 zeigt den Kundenpfad eines abfliegenden Passagiers im Überblick. Als besonders relevant erachtet wurden von den Experten und Kunden folgende Kontaktpunkte: Check-in, Passkontrolle, Security, Gastronomie, Shopping, 


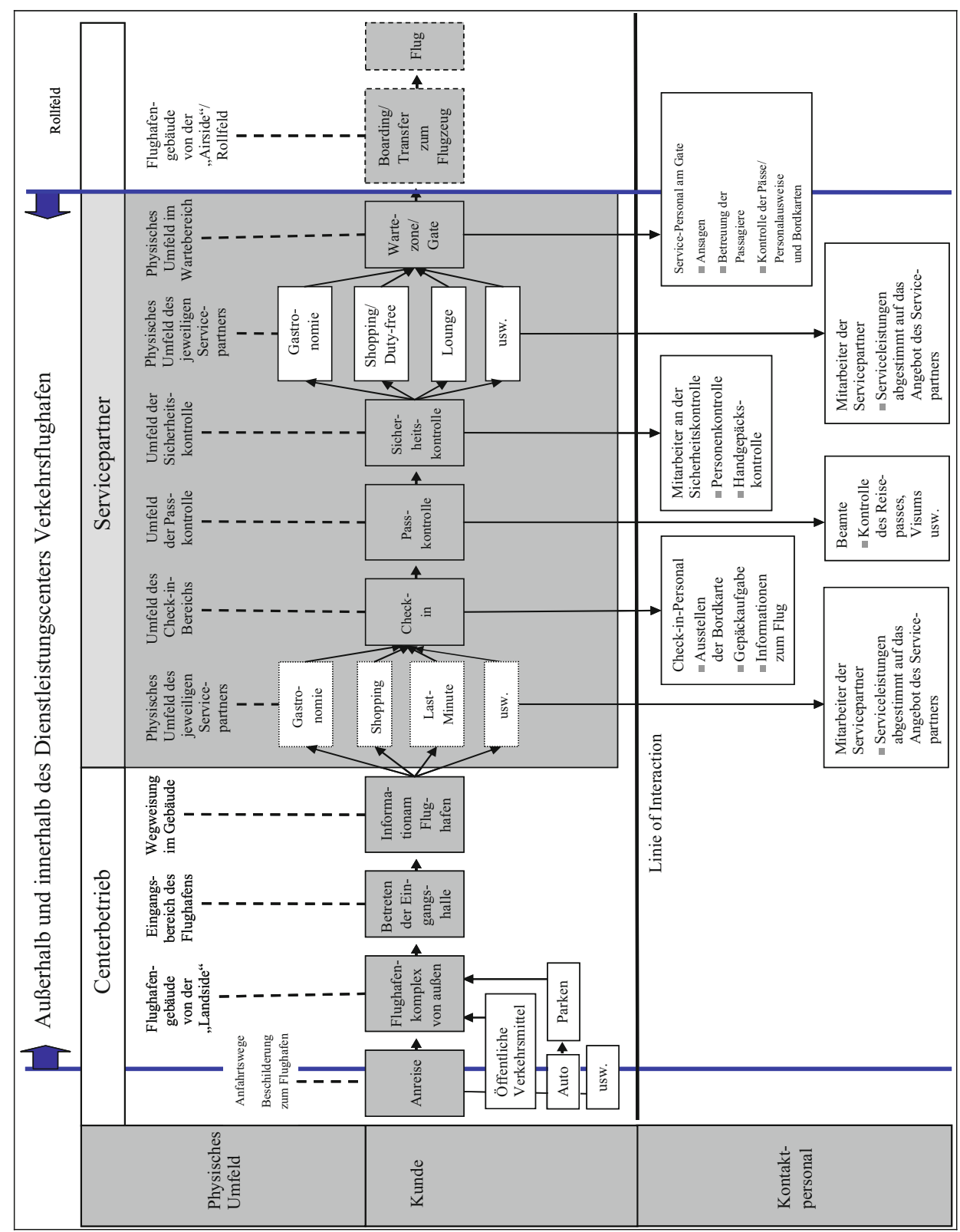

Abb. 4: Blueprint des Kundenpfades eines abfliegenden Passagiers

Lounge und Wartebereich. Mit Ausnahme der Lounge wurden alle diese Kontaktpunkte in der weiteren Untersuchung berücksichtigt. Die Lounge konnte in die Untersuchung nicht mit einbezogen werden, da eine Befragung zur Qualität dieses Kontaktpunktes sowohl in als auch vor der Lounge untersagt wurde. 


\subsection{Operationalisierung der Konstrukte}

\subsubsection{Centerbetriebsqualität und Kontaktpunktqualitäten}

Bei der Centerbetriebsqualität und den Kontaktpunktqualitäten handelt es sich um nicht direkt beobachtbare Variablen, so genannte hypothetische Konstrukte. Daher gilt es im Folgenden, die Messindikatoren der Modellkonstrukte zu identifizieren.

Im Rahmen der Operationalisierung der Centerbetriebsqualität und der Kontaktpunktqualitäten erfolgt die Orientierung am C-OAR-SE-Verfahren zur Konstruktoperationalisierung (vgl. zur C-OAR-SE-Prozedur Rossiter 2002) ${ }^{8}$ Zunächst wird eine genaue Definition und Spezifikation der Konstruktinhalte vorgenommen sowie - mittels Experten- und Kundeninterviews - die Sammlung möglichst vieler Indikatoren, um alle Konstruktfacetten abdecken zu können. Letzteres wird zur Sicherstellung der Inhaltsvalidität angestrebt. Folgende Vorgehensweise im Rahmen der Interviews wurde dabei gewählt:

Zunächst wurde den Experten die Aufgabe gestellt, den Kundenpfad eines abfliegenden Passagiers und die darin enthaltenen Kontaktpunkte näher zu beschreiben (vgl. Abschn. 4.1). Als Experten wurden Mitarbeitende des Flughafenmanagements und der Fluggesellschaften angesehen, da von diesen gute Kenntnisse der Kundenprozesse an einem Flughafen erwartet wurden. Die so identifizierten Kundenkontaktpunkte dienten zur Strukturierung des weiteren Interviews. Die Befragten wurden - ohne weitere Hilfestellung - gebeten, nacheinander für jeden der Kundenkontaktpunkte die für einen guten Qualitätseindruck kritischen, qualitätsbeeinflussenden Merkmale aus Passagiersicht zu benennen. Im Anschluss daran wurde den Interviewpartnern die Aufgabe gestellt, weitere Qualitätsmerkmale aufzuzählen, die neben der Kontaktpunktkette einen Einfluss auf die Qualitätswahrnehmung des Flughafens ausüben. Damit wurde der Zielsetzung Rechnung getragen, dass neben einzelnen Servicepartnern der Centerbetrieb des Flughafens Einfluss auf das Qualitätsurteil des Kunden nimmt. Um sicherzustellen, dass eine Vielzahl von Aspekten im Rahmen der Interviews zur Sprache kamen, erfolgte ein Nachfragen hinsichtlich der in den Abschn. 3.1 und 3.2 identifizierten Qualitätsdimensionen, sowohl für die einzelnen Kontaktpunkte als auch für den Centerbetrieb.

Da die Qualität des Dienstleistungscenters Flughafen später aus Kundensicht gemessen werden sollte, wurden ferner Passagierinterviews im Wartebereich des Flughafens geführt. Ausgehend von der Schilderung der vom Passagier angelaufenen Kontaktpunkte und einer Visualisierung in einem kundenspezifischen Blueprint stand auch hier die Ermittlung von Qualitätsmerkmalen im Mittelpunkt. Um die Befragten nicht zu überfordern und den Passagieren die Möglichkeit zu geben, sich alle qualitätsbeeinflussenden Aspekte der einzelnen Service Encounter wieder ins Gedächtnis zu rufen, kam hierbei eine gestaffelte auf der Sequentiellen Ereignismethode (Sequential Incident Method) ${ }^{9}$ aufbauenden Vorgehensweise zum Einsatz. Die Passagiere wurden anhand des unter ihrer Mitwirkung erstellten Ablaufdiagramms schrittweise durch die einzelnen Kontaktpunkte geführt und um eine Schilderung positiver und negativer Erlebnisse gebeten. Dabei wurden negative Erlebnisberichte gleichzeitig dafür genutzt, vom Probanden Verbesserungsvorschläge zu erhalten. Erst im Anschluss an die Schilderung der jeweiligen Kontaktsituation erfolgte die Bitte, zentrale Qualitätsaspekte für den jeweiligen Kontaktpunkt zu benennen. Entsprechend der Vorgehensweise bei den Experteninterviews wurde anschließend nach wei- 
teren Merkmalen gefragt, die neben der „Kontaktpunktkette“ die Qualitätswahrnehmung des Flughafens an sich beeinflussen. Auch hier wartete der Interviewer ab, zu welchen Qualitätsdimensionen eine Aussage getroffen wurde und fragte dann bezüglich fehlender Dimensionen nach.

Die gesammelten Qualitätsmerkmale zu jedem Kontaktpunkt sowie zum Centerbetrieb wurden anschließend von zwei Mitarbeitenden des Flughafenmanagements und sechs Passagieren nochmals auf Vollständigkeit, Redundanz und Verständlichkeit überprüft. Die Mitarbeitenden des Flughafenmanagements wurden in Einzelinterviews um eine Überprüfung der Merkmalslisten gebeten, während die Passagiere im Rahmen von Fokusgruppeninterviews eine Überprüfung vornahmen. Ferner wurden auf Empfehlung des Vorgehens von Rossiter und aus inhaltlichen Überlegungen heraus einige Items eliminiert (Rossiter 2002, S. 314). Bei den eliminierten Items handelt es sich um Indikatoren, die entweder bereits durch andere Indikatoren abgedeckt sind oder um solche, die die Befragten als inhaltlich nicht zum zu messenden Konstrukt passend betrachteten. Mit diesem Vorgehen soll zum einen einer zu hohen Multikollinearität vorgebeugt und zum anderen eine möglichst hohe Inhaltsvalidität sichergestellt werden.

Die Centerbetriebsqualität sowie die Kontaktpunktqualitäten werden in der vorliegenden Untersuchung formativ operationalisiert, da die betreffenden Indikatoren als verantwortlich für die Bildung und Veränderung des Konstrukts angesehen werden. ${ }^{10}$ Tabelle 1 und 2 im Anhang geben einen Überblick über die Messitems der Centerbetriebsqualität und der Kontaktpunktqualitäten. Wie daraus ersichtlich wird, werden mit Ausnahme des Bereichs technische Ausstattung sämtliche im Abschn. 3.1 vorgestellten Bereiche der Centerbetriebsqualität durch die identifizierten Indikatoren abgedeckt. ${ }^{11}$ Ferner zeigt sich, dass sich sämtliche Indikatoren der Kontaktpunktqualitäten den Bereichen Interaktion und/oder Leistung zuordnen lassen. Der Bereich Interaktion enthält dabei Merkmale, die in Zusammenhang mit der Serviceorientierung (wie z. B. Freundlichkeit) stehen. Der Bereich Leistung umfasst Merkmale, die Bezug zum Leistungsumfeld (z. B. Einrichtung, Atmosphäre) und zu den Leistungsfaktoren nehmen (z. B. Merkmale, die mit der Leistungserstellung korrespondieren wie die Auswahl an Speisen). Damit bestätigt sich die im Abschn. 3.2 aufgestellte Vermutung, nach der sich die Wahrnehmung der Kontaktpunktqualitäten zum einen an der Interaktion zwischen dem Centernutzer und dem Kontaktpersonal der Servicepartner und zum anderen an leistungsbezogenen Aspekten orientiert.

\subsubsection{Dienstleistungscenterqualität}

Bei der Auswahl der Messindikatoren der Dienstleistungscenterqualität ist zunächst auf die große Ähnlichkeit zwischen den Konstrukten Kundenzufriedenheit und Dienstleistungsqualität hinzuweisen. Dies verdeutlicht bereits das Begriffsverständnis der Kundenzufriedenheit. So wird nach dem in der Literatur dominanten Confirmation-DisconfirmationParadigma die Zufriedenheit eines Kunden als das Resultat eines komplexen Prozesses der Informationsverarbeitung verstanden, im Rahmen dessen die aktuellen Erfahrungen, die mit einem Anbieter gemacht werden, mit (früheren) Erwartungen bzw. einem Anspruchsniveau oder Ideal verglichen werden (Oliver 1977, 1996, S. 98 ff. und 1997). Ein positiver Erwartungs-Beurteilungs-Vergleich löst demnach Zufriedenheit und ein negativer Vergleich Unzufriedenheit aus. 
Zur Unterscheidung der beiden Konstrukte wird angeführt, dass bei der Dienstleistungsqualität die Kundenerwartungen im Hinblick auf einzelne Leistungsmerkmale bei der Beurteilung dieser Merkmale eine Rolle spielen, wohingegen bei der Kundenzufriedenheit der Grad der Erfüllung der Kundenerwartungen, wie er vom Kunden insgesamt empfunden wird, im Vordergrund steht. Entgegen dieser Auffassung wird im Folgenden diese Unterscheidung nicht gemacht, sondern die Dienstleistungscenterqualität als globale Zufriedenheit mit dem Dienstleistungscenter modelliert. Begründen lässt sich dies damit, dass die Erfüllung der Kundenerwartungen bezüglich der einzelnen Leistungsmerkmale bereits mit den jeweiligen Teilqualitäten gemessen wird. Somit wird hier mit der Dienstleistungsqualität der Abgleich mit den Kundenerwartungen insgesamt, d. h. über die einzelnen Leistungsmerkmale hinweg, gemessen.

Im Gegensatz zur Centerbetriebsqualität sowie den Kontaktpunktqualitäten, die noch nicht bzw. nicht in diesem Kontext untersucht wurden, kann für die Messung der Dienstleistungscenterqualität auf bestehende Skalen zurückgegriffen werden. Im Rahmen der vorliegenden Untersuchung erfolgt vorwiegend eine Orientierung an Items, die eine empirisch bewährte Basis für die Messung der Kundenzufriedenheit bilden (Fornell et al. 1996; Homburg u. Fürst 2008). Item DLCQ_1 ist selbst konstruiert. Eine Bestimmung der Dienstleistungscenterqualität mittels der vier Indikatoren hat den Vorteil, dass sie, z. B. durch die Ermittlung der wahrgenommenen Kongruenz mit dem Ideal, ein umfassenderes Bild bietet als eine Ermittlung der Dienstleistungscenterqualität mittels eines Indikators. Ferner wird eine reflektive Operationalisierung vorgenommen. Dies basiert auf der Überlegung, dass in diesem Fall die Veränderung des Konstruktes eine Veränderung sämtlicher Indikatoren bewirkt. Tabelle $3 \mathrm{im}$ Anhang gibt einen Überblick über die verwendeten Items.

Im Anschluss an die Operationalisierung der Konstrukte widmet sich der folgende Abschnitt der empirischen Analyse des Qualitätsmodells für Dienstleistungscenter. Die Datenerhebung fand im Wartebereich des Flughafens statt. Als Datenerhebungsmethode diente eine schriftliche Befragung. Diese stützte sich auf einen standardisierten Fragebogen. Die Fragen wurden mit einer zweipoligen, fünfstufigen Ratingskala verbunden. Als Extrema wurden für die Statements die Formulierungen „trifft überhaupt nicht zu“ bis „trifft voll und ganz zu“ gewählt. Insgesamt wurden im Befragungszeitraum 1.542 Passagierdatensätze generiert (bei einer Verweigerungsquote von 8 Prozent). Die Mehrheit der Probanden waren - mit fast identischen Anteilen - Schweizer, Deutsche und Franzosen. Im Hinblick auf Geschlecht und Alter ist die Stichprobe länderübergreifend annähernd repräsentativ.

\subsection{Schätzung des Kausalmodells und Modellbeurteilung}

Zur Schätzung von Kausalmodellen existieren zwei verschiedene Verfahren. Zum einen das Verfahren der Kovarianzstrukturanalyse (Linear Structural Relationships), das vor allem durch die Softwareanwendung AMOS unterstützt wird sowie zum anderen der varianzbasierte Partial-Least-Squares (PLS)-Ansatz, der insbesondere mit Hilfe der Software PLS-Graph und Smart-PLS Anwendung findet (Ringle 2004b; Albers u. Hildebrandt 2006). In der Marketingforschung wird zunehmend Rekurs auf den Partial-Least-Squares (PLS-)-Ansatz genommen, da dieser im Gegensatz zu den kovarianzbasierten Verfahren 
insbesondere auch dann zur Anwendung kommen kann, wenn die Multinormalverteilung der manifesten Variablen nicht gegeben ist, die Stichprobe relativ klein ist und formative Konstrukte zu berücksichtigen sind (Albers u. Hildebrandt 2006). ${ }^{12}$ Für welches der beiden Verfahren der Strukturgleichungsanalyse sich zu entscheiden ist, hängt letztlich vom Anliegen des Forschers ab. ${ }^{13}$ Liegt der Fokus der Untersuchung in der bestmöglichen Erklärung der Veränderung einer oder mehrerer Zielvariablen für die Identifikation von zentralen Determinanten, so bietet sich der Rückgriff auf ein varianzbasiertes Verfahren an. Besteht das Anliegen des Forschers jedoch darin, ein neuartiges, theoriebasiertes Hypothesengefüge zwischen latenten Konstrukten zu untersuchen, so ist der Einsatz kovarianzbasierter Verfahren zu favorisieren (Herrmann et al. 2006). Da im vorliegenden Fall die Ermittlung von Einflussfaktoren auf die Dienstleistungscenterqualität im Mittelpunkt steht, wurde sich im vorliegenden Artikel für die Anwendung eines varianzbasierten Verfahrens entschieden. Die Strukturgleichungsanalyse erfolgt dabei auf Basis des Analyseprogramms PLS Graph 3.0 (Chin 2003).

Zur Beurteilung von varianzbasierten PLS-Ergebnissen liegt noch kein geeignetes globales Gütemaß vor. Zur Abschätzung der Modellgüte, und damit zur Sicherstellung der Zuverlässigkeit des Gesamtmodells, erfolgt daher eine Orientierung an den von Chin vorgeschlagenen Prüfkriterien (Chin 1998b, S. 316 ff.; Chin u. Newsted 1999, S. 312; vgl. für eine Übersicht über die Prüfkriterien für PLS-Modelle, Herrmann et al. 2006, S. 61). Dabei sind die formativen und reflektiven Messmodelle sowie das Strukturmodell getrennt voneinander zu beurteilen (vgl. zu den Ergebnissen der Messmodelle Tab. 4 im Anhang).

Bei formativen Messmodellen sind insbesondere die multiplen Regressionskoeffizienten zwischen dem Konstrukt und den Indikatoren sowie deren Signifikanz von Interesse. Diese messen als Gewichte den Einfluss der Indikatorvariablen auf die zugehörige latente Variable. Die Höhe der Regressionskoeffizienten ermöglicht eine Aussage über die Vorhersagevalidität eines Indikators im Hinblick auf das Konstrukt. Der dazugehörige TWert liefert Anhaltspunkte zur Einschätzung der Reliabilität des Regressionskoeffizienten (Herrmann et al. 2006, S. 61). Bezüglich des T-Wertes sind in der vorliegenden Studie für die einzelnen Konstrukte unterschiedliche Resultate festzustellen. Während sämtliche Indikatoren des Konstruktes Check-in-Qualität signifikant sind, verfehlt ein Großteil der Items der Konstrukte Shopping-, Gastronomie- und Wartebereichsqualität den erforderlichen T-Wert von 1,98. Auffallend bei Letzteren ist, dass Leistungsfaktoren wie z. B. Vielfalt des Warenangebots (Shop_3), Auswahl an Speisen (Rest_3) oder Zeitschriftenangebot (Wartb_5) kaum eine Bedeutung zukommt, demgegenüber aber Items, die dem Leistungsumfeld zugehören, wie z. B. Entspannungsmöglichkeit im Restaurant/in der Bar, Annehmlichkeit des Restaurant-/Barbesuchs bzw. des Besuchs im Geschäft oder Atmosphäre im Wartebereich (Rest_5, Rest_6, Shop_4, Wartb_6), einen hohen Einfluss auf die latente Variable ausüben. Die Indikatorgewichte und T-Werte der Konstrukte Check-in-, Passkontrollen- und Security-Qualität offenbaren, dass insbesondere jene Indikatorvariablen, die in Zusammenhang mit der Einrichtung der jeweiligen Bereiche (Check_3, Pass_2, Sich_3) sowie mit der Freundlichkeit des Personals (Check_1, Pass_1, Sich_1) stehen, für die Bildung der Kontaktpunktqualitäten von Bedeutung sind. Wichtige Indikatoren für die Centerbetriebsqualität stellen das Sicherheitsempfinden der Centernutzer (CBQ_8), die Großzügigkeit des Flughafengebäudes (CBQ_4) und die Vielfalt der angeflogenen Desti- 
nationen (CBQ_9) dar. Mit Hilfe des Variance Inflation Factors lässt sich außerdem die Multikollinearität zwischen den formativen Indikatoren untersuchen. Dieser basiert auf dem Varianzanteil eines Indikators, der durch die übrigen Konstruktindikatoren erklärt werden kann. Liegt eine hohe Multikollinearität vor, führt dies zum einen dazu, dass die Schätzung der Regressionskoeffizienten nicht stabil ist, und zum anderen, dass die Indikatoren redundante Informationen enthalten (Riemenschneider 2005). Im vorliegenden Fall liegen die Varianzinflationswerte (VIF-Werte) für sämtliche Indikatoren weit unter dem Höchstwert von 10, somit liegt keine zu hohe Multikollinearität vor (siehe Tab. 5 im Anhang). Im Unterschied zu reflektiven Messmodellen, kann die Untersuchung der internen Konsistenz bei formativ gemessenen Konstrukten aufgrund der nicht notwendigen Abhängigkeit zwischen den Indikatoren eines Konstrukts nicht erfolgen. Daher wird die Messung der externen Validität empfohlen (Diamantopoulos u. Winklhofer 2001; Jarvies et al. 2003). Damit wird überprüft, ob die formativen Indikatoren das Konstrukt angemessen abbilden. Aufschluss über die externe Validität gibt die Einführung einer Phantomvariablen. Im vorliegenden Fall werden die formativen Konstrukte durch je eine Phantomvariable mit zwei Indikatoren reflektiv abgebildet und mit dieser in Verbindung gesetzt. Die Indikatoren dieser Variablen erfassen die Gesamtqualität des jeweiligen Kontaktpunktes bzw. der Centerbetriebsqualität durch den Kunden. Es zeigt sich, dass zwischen sämtlichen Konstrukten und der Phantomvariable ein starker und signifikanter Zusammenhang besteht und ein erheblicher Teil der Varianz der Phantomvariablen durch das jeweilige Konstrukt erklärt werden. Somit kann auf externe Validität der formativen Messmodelle geschlossen werden (vgl. hierzu ausführlich Diamantopoulos u. Winklhofer 2001, S. 273; Götz u. Liehr-Gobbers 2004, S. 22).

Im Hinblick auf das reflektive Messmodell des Konstrukts Dienstleistungscenterqualität sind durchgehend positive Resultate festzustellen. Von Item DLCQ_1 abgesehen, liegen sämtliche Faktorladungen des Konstrukts deutlich über einem Wert von 0,8. ${ }^{14}$ Die dazugehörigen T-Werte übersteigen stets den geforderten Mindestwert von 1,64. Ferner beträgt die durchschnittlich erfasste Varianz 0,726 und übersteigt somit klar den geforderten Wert von 0,5. Ein Cronbach'sches Alpha von 0,909 sowie der Wert für die Konstruktreliabilität $(0,914)$ verweisen zudem auf das Vorliegen von hoher interner Konsistenz.

Im Rahmen der Beurteilung des Strukturmodells interessiert die Erklärung der Zielvariablen, hier der Dienstleistungscenterqualität, durch die exogenen Variablen. Dies erfolgt über das $\mathrm{R}^{2}$, das den durch die latenten exogenen Variablen erklärten Varianzanteil angibt. Die Varianz der Dienstleistungscenterqualität setzt sich zu 42,2 Prozent aus den Kontaktpunktqualitäten sowie der Centerbetriebsqualität zusammen. Dieser Wert ist für PLS-Modelle als „,mittelgut“ bis „,substanziell“ anzusehen (Chin 1998b, S. 323). Mit dem Stone-Geisser-Kriterium steht ein Gütekriterium für die Vorhersagevalidität des Modells zur Verfügung. Die Bestimmung des Stone-Geisser-Testkriteriums $Q^{2}$ ergibt im vorliegenden Fall für sämtliche latenten endogenen Variablen einen Wert größer Null, d. h., dem Strukturmodell kann insgesamt Vorhersagerelevanz zugesprochen werden. Als weiteres Gütekriterium kann über die Effektstärke $f^{2}$ eine Aussage darüber getroffen werden, ob durch ein einzelnes exogenes Konstrukt ein zusätzlicher Erklärungsgewinn hinsichtlich des endogenen Konstrukts erreicht wird. Die Effektstärke gibt an, inwieweit durch die Berücksichtigung der betrachteten exogenen Variablen im Strukturmodell die erklärte Varianz der entsprechenden endogenen Variablen erhöht werden kann (Götz u. Liehr-Gobbers 


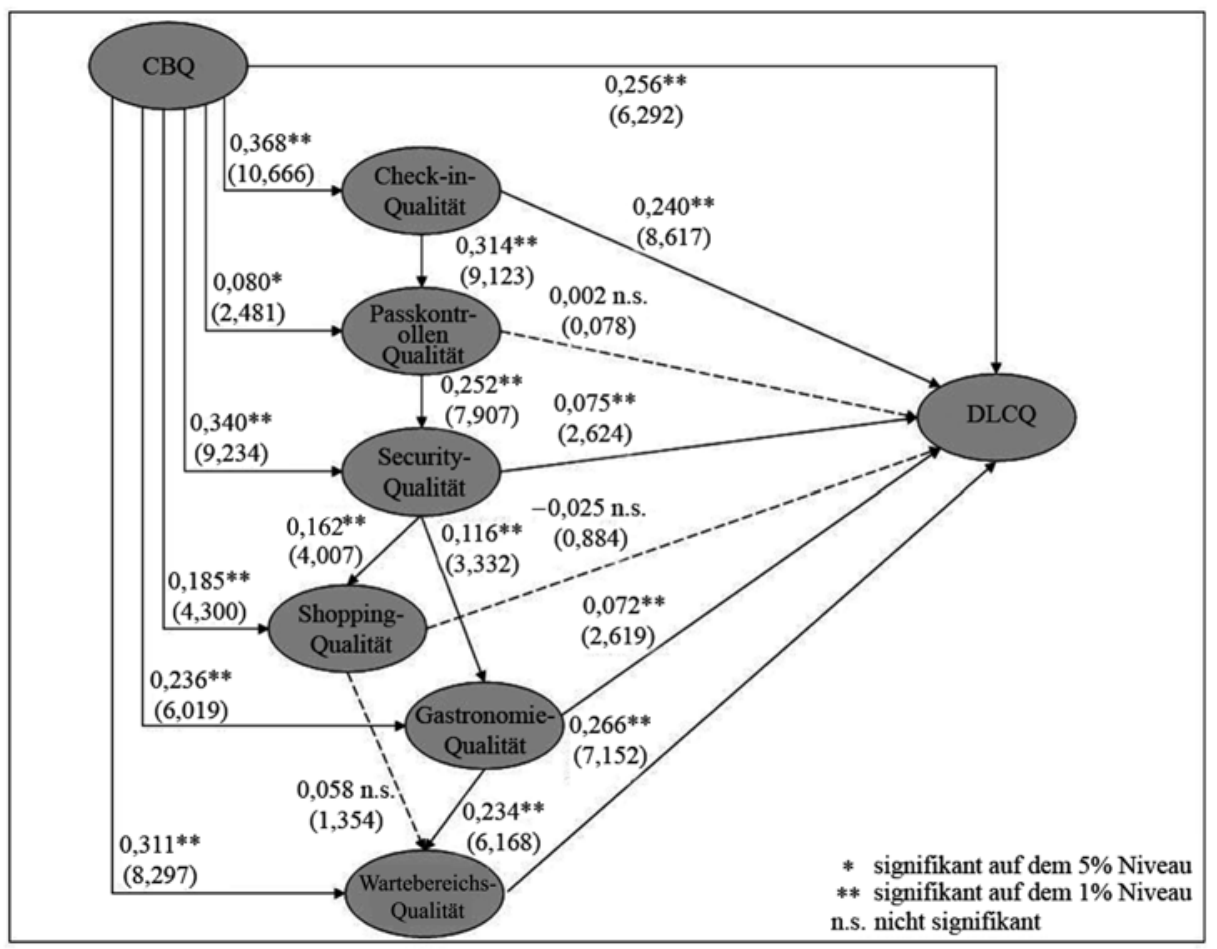

Abb. 5: Beziehungszusammenhänge im Kausalmodell zur Dienstleistungscenterqualität

2004). Die Ergebnisse offenbaren, dass die Wartebereichsqualität (0,095), gefolgt von der Centerbetriebsqualität $(0,085)$ und der Check-in-Qualität $(0,073)$, mit Abstand den größten Einfluss auf die Dienstleistungscenterqualität hat. Dies bestätigt die vergleichsweise hohe Relevanz der Variablen.

Im Rahmen des Strukturgleichungsmodells erfolgt schließlich eine Überprüfung der Hypothesen über die Wirkungsbeziehungen (vgl. hierzu ausführlich Abschn. 4.4). Die Pfadkoeffizienten geben Aufschluss über die Einflussstärke eines Konstrukts auf ein kausal nachfolgendes. Um deren Güte abzuschätzen, wird erneut auf die Signifikanzen auf Basis der T-Werte zurückgegriffen. Abb. 5 liefert einen Überblick über die Beziehungszusammenhänge im Qualitätsmodell sowie die dazugehörigen T-Werte (in Klammer).

\subsection{Interpretation der Ergebnisse}

Basierend auf der kausalanalytischen Prüfung des Qualitätsmodells lassen sich folgende Ergebnisse ableiten: Die vom Kunden wahrgenommene Centerbetriebsqualität hat einen direkten positiven und auf dem 1\%-Niveau signifikanten Einfluss auf die Dienstleistungscenterqualität. Damit bestätigt sich Hypothese $\mathrm{H}_{1}$, dass die Centerbetriebsqualität als bewertete Beschaffenheit aller centerbetriebsspezifischen Rahmenfaktoren auf das Gesamtqualitätsurteil des Dienstleistungscenters Einfluss nimmt. Beachtenswert ist hierbei 
die Effektstärke. Sie fällt mit einem Wert von 0,256 meist stärker aus als die Wirkungsbeziehungen zwischen den übrigen Teilqualitäten und der Dienstleistungscenterqualität. Dementsprechend spielt die Wahrnehmung der Centerbetriebsqualität durch die Passagiere eine zentrale Rolle für die Beurteilung des gesamten Dienstleistungscenters.

Hinzu kommt, dass sich sämtliche postulierten Wirkungsbeziehungen zwischen der Centerbetriebsqualität und den einzelnen Kontaktpunktqualitäten (Hypothese $\mathrm{H}_{2}$ ) bestätigen. Am stärksten wird die Check-in-Qualität mit einer Pfadhöhe von 0,368, gefolgt von der Security-Qualität $(0,340)$ und Wartebereichsqualität $(0,311)$, durch die Centerbetriebsqualität beeinflusst. Es kann vermutet werden, dass beim Betreten des Flughafens und beim Warten am Check-in die Rahmenfaktoren, die den Centerbetrieb ausmachen, wie z. B. Architektur und Design, für den Centernutzer sehr präsent sind. Ähnliches kann für den Wartebereich angenommen werden. Auch hier hält sich der Passagier längere Zeit auf. Ferner ist der starke Einfluss auf die Security-Qualität auf Rahmenfaktoren wie z. B. das Sicherheitsempfinden zurückzuführen.

Ein sehr geringer Beeinflussungseffekt und im Vergleich zu den anderen Konstrukten weniger signifikanter Einfluss besteht für die Passkontrollen-Qualität. So liegt eine Pfadhöhe von 0,080 auf dem 5\%-Signifikanzniveau vor). Es kann angenommen werden, dass die vergleichsweise geringe Aufenthaltsdauer hierbei eine Rolle spielt. Die behördlichen Leistungen werden von den Passagieren vielfach als ein „,notwendiges Übel“ betrachtet. Der Fokus liegt auf dem schnellen Durchlaufen der Kontrolle. Dem institutionellen Rahmen, in den die Passkontrolle eingebettet ist, wird daher kaum Aufmerksamkeit geschenkt.

Die empirischen Ergebnisse zeigen weiterhin, dass nicht alle Kontaktpunktqualitäten direkten Einfluss auf das Gesamtqualitätsurteil bzgl. des Dienstleistungscenters nehmen. Damit ist die Hypothese $\mathrm{H}_{3}$ aufgrund des nicht existenten Zusammenhangs zwischen Passkontrollen- bzw. Shopping-Qualität und der Dienstleistungscenterqualität abzulehnen. Auffallend ist der im Vergleich zu den übrigen Kontaktpunkten starke Einfluss des ersten Kontaktpunktes, der Check-in-Qualität $(0,240)$, und des letzten Kontaktpunktes, der Wartebereichs-Qualität (0,266). Dies lässt sich auf den Primacy- und Recency-Effekt zurückführen. Diesen zufolge bleiben die zuerst bzw. zuletzt gebotenen Informationen - in diesem Fall Kontaktpunkte - besser in Erinnerung als die übrigen Informationen (Kontaktpunkte) (vgl. hierzu ausführlich Haugtvedt u. Wegener 1994, S. 206 ff.). Aus diesem Grund ist es sinnvoll, der Planung und der Gestaltung der Qualität - aber auch der Qualitätskontrolle - an diesen Kontaktpunkten besondere Beachtung zu schenken.

Die Betrachtung der Dienstleistungssequenz - bestehend aus den einzelnen Kontaktpunkten - bestätigt im Unterschied zu den Studien von Bauer et al. (2000) und Danaher u. Mattson (1994a, 1994b) nur teilweise die direkte positive Einflussnahme des Qualitätsurteils bzgl. eines Kontaktpunktes auf die qualitative Wahrnehmung des darauf folgenden Kontaktpunktes. Hypothese $\mathrm{H}_{4}$ ist demnach abzulehnen. Hinsichtlich des Wirkungszusammenhangs der einzelnen Beziehungen sind Unterschiede feststellbar. Während der Wirkungszusammenhang von der Shopping-Qualität auf die Wartebereichsqualität sehr gering und zudem nicht signifikant ist, besteht mit einem Wert von 0,314 ein starker direkter und signifikanter Effekt von der Check-in-Qualität auf die Passkontrollenqualität. Aufgrund der Werte im vorliegenden Fall kann vermutet werden, dass sich Kontaktpunkte, die in inhaltlichem Zusammenhang stehen (wie z. B. Check-in und Passkontrolle) hinsichtlich ihrer Qualitätswahrnehmung stärker beeinflussen als unähnliche Kontaktpunkte. 


\section{Zusammenfassung und Implikationen}

Der Zusammenschluss in Form eines Dienstleistungscenters stellt besondere Anforderungen an die Qualitätsmessung. Daher war es das Ziel des Beitrages, auf Basis theoretischer Überlegungen ein Modell zur Messung der Qualität in Dienstleistungscentern zu entwickeln. Um diese Zielsetzung zu erreichen, erfolgte zunächst eine theoriegeleitete Identifikation der Einflussfaktoren der Dienstleistungscenterqualität. Darauf aufbauend wurde eine Definition der Dienstleistungscenterqualität entwickelt. Schließlich stand die Entwicklung eines Modells, das die Entstehung der Dienstleistungsqualität abbildet, im Vordergrund.

Auf Basis der theoretischen Überlegungen sind folgende zentrale Erkenntnisse festzuhalten: Der Centerbetrieb bildet den institutionellen Rahmen für die innerhalb des Dienstleistungscenters angesiedelten Servicepartner. Er untersteht der Leitung des Centermanagements mit eigenem Personal, den Gebäude- und Flächeneinheiten sowie den zugehörigen Ausstattungs- und Einrichtungselementen. An jedem der Kontaktpunkte gewinnt der Kunde zudem einen Eindruck über die Leistungen eines Servicepartners. Die Qualitätsbeurteilung durch den Centernutzer bildet sich somit zum einen auf Basis von Einzelinteraktionen (im Sinne von Kontaktpunkten) mit verschiedenen Servicepartnern und zum anderen auf Basis bestehender institutioneller Rahmenbedingungen (d. h. dem Centerbetrieb). Das globale Qualitätsurteil bezüglich des Dienstleistungscenters eines Centernutzers stellt damit eine Funktion aus n Kontaktpunktqualitäten (mit $\mathrm{k}=1$ bis $\mathrm{n}$ ) und der Centerbetriebsqualität dar.

Basierend auf den Ergebnissen der beispielhaft durchgeführten empirischen Überprüfung ergeben sich folgende Erkenntnisse: Die Centerbetriebsqualität sowie der erste und der letzte Kontaktpunkt, den ein Centernutzer durchläuft (in diesem Falle die Check-inund Wartebereichsqualität), haben einen besonders starken Einfluss auf das Gesamturteil des Kunden bezüglich des Dienstleistungscenters. Zwischen der Passkontrollen- bzw. Shopping-Qualität und der Dienstleistungscenterqualität besteht hingegen kein signifikanter Zusammenhang. Wichtige Qualitätstreiber stellen bei der Centerbetriebsqualität das Sicherheitsempfinden der Centernutzer, die Großzügigkeit des Flughafengebäudes sowie die Vielfalt der angeflogenen Destinationen dar. Bei den Kontaktpunkt-Qualitäten sind insbesondere Merkmale, die in Zusammenhang mit dem Leistungsumfeld stehen, von Bedeutung (z. B. Einrichtung des Check-in- bzw. Sicherheitsbereichs, Komfort der Sitzmöglichkeiten und Atmosphäre im Wartebereich).

Aus diesen Erkenntnissen lassen sich folgende Implikationen ableiten: Es ist ein Qualitätsmanagementsystem einzuführen, das die Phasen des klassischen Managementprozesses beinhaltet: Analyse, Planung, Umsetzung bzw. Gestaltung und Kontrolle. Im Rahmen der Analysephase ist entsprechend der Vorgehensweise in Kap. 3 und 4 ein Qualitätsmodell für den betrachteten Dienstleistungscenter zu entwickeln. Hierbei sind die einzelnen Teilqualitäten zu identifizieren, die als Basis zur Beurteilung der Dienstleistungscenterqualität aus Kundensicht herangezogen werden können. In der Planungsphase gilt es, die Ziele des Qualitätsmanagements festzulegen und centerbetriebs- sowie servicepartnerbezogene Qualitätsstrategien abzuleiten, und so die qualitätsbezogene strategische Ausrichtung des Dienstleistungscenters festzulegen. Um die gesetzten Qualitätsziele zu erreichen, werden in der Phase der Qualitätsgestaltung qualitätssteigernde Maßnahmen eingesetzt. Dabei hat das Centermanagement neben Qualitätsverbesserungsmaßnahmen, die am Centerbetrieb 
ansetzen, auch die Möglichkeit, auf die Qualitätsgestaltung der Servicepartner Einfluss zu nehmen. Schließlich ist im Rahmen der Qualitätskontrollphase zu überprüfen, inwiefern die Qualitätsziele erreicht wurden.

Für den untersuchten Dienstleistungscenter „Flughafen“ ergibt sich folgende Konkretisierung von Implikationen: Der Centerbetrieb hat dafür Sorge zu tragen, das Sicherheitsempfinden der Centernutzer positiv zu beeinflussen. Dies kann beispielsweise durch Polizeipräsenz oder die Ausweitung der Videoüberwachung des Flughafengeländes bei gleichzeitiger Anbringung von Hinweisschildern erfolgen. Ferner sind Maßnahmen zu ergreifen, die das physische Erscheinungsbild des Centers verbessern. Hierzu sind nachträgliche Veränderungen der Innen- und Außenarchitektur sowie Anpflanzungen im Umfeld sowie „Begrünungen“ innerhalb des Centers denkbar. Schließlich hat das Centermanagement darauf zu achten, dass das Leistungsangebot auf die Bedürfnisse der Centernutzer zugeschnitten ist. Für das Leistungsangebot eines Flughafens kann dies dahingehend spezifiziert werden, dass eine ausreichende Anzahl an Destinationen durch Verhandlungen mit bestehenden bzw. potentiellen Fluggesellschaften gewährleistet wird sowie für den Kunden attraktive Fluggesellschaften am Flughafen vertreten sind. Der Centerbetrieb hat auch die Möglichkeit, auf die Qualitätsgestaltung der Servicepartner Einfluss zu nehmen. Die erste Möglichkeit besteht hierbei über die Akquisition und Selektion geeigneter Partnerunternehmen. Hierzu ist ein differenzierter Kriterienkatalog zu entwerfen, anhand dessen die Eignung der potenziellen Servicepartner bewertet wird. Als Leitfaden können hierzu die als relevant identifizierten Qualitätsmerkmale der zugehörigen Kontaktpunkte dienen. Die zweite Möglichkeit der Einflussnahme setzt in der „Anbindungs- und Integrationsphase" an. Hier kann das Centermanagement durch die Ausgestaltung von Verträgen mit den ausgewählten Servicepartnern versuchen, die erwartete Dienstleistungsqualität sicherzustellen.

Es ist darauf hinzuweisen, dass die Problematik, die mit der Messung der Dienstleistungsqualität verbunden ist, mit diesem Beitrag keineswegs abschließend behandelt wurde. Vielmehr unterliegt dieser Artikel auch einigen Restriktionen.

So erfolgte eine Einschränkung auf dyadisch-persönliche Kundenkontaktpunkte als Einflussfaktoren der Dienstleistungscenterqualität. Für weitere Forschungsarbeiten wäre es interessant, Interaktionen mit einzubeziehen, in denen der Kunde mit „Technik“ interagiert. Beispiele hierfür sind Self-Check-in-Schalter, Essens- und Getränkeautomaten, Bankomaten. Es ist davon auszugehen, dass sich die im vorliegenden Artikel analysierten Kontaktpunktqualitäten zwischenmenschlicher Interaktionen hinsichtlich ihrer Indikatoren deutlich von Kontaktpunktqualitäten, die auf Mensch-Technik-Interaktionen basieren, unterscheiden.

Für zukünftige Untersuchungen erscheint darüber hinaus die Berücksichtigung verhaltensbezogener Größen als Folge der Dienstleistungscenterqualität notwendig. Durch die Verknüpfung des ökonomischen Erfolgs mit Größen auf der Wirkungsseite der Dienstleistungscenterqualität kann im Rahmen von empirischen Untersuchungen der Beitrag einzelner qualitätssteigernder Maßnahmen zum ökonomischen Erfolg ermittelt werden.

Die empirische Untersuchung beschränkte sich außerdem auf den Dienstleistungscenter „Flughafen“. Daher ist das entwickelte Qualitätsmodell auch anhand anderer Dienstleistungscenter (z. B. Medical-, Shopping-, Freizeit-, Bürocenter) zu überprüfen. Dabei ist von Interesse, inwieweit sich das entwickelte Qualitätsmodell an andere Gegebenheiten anpassen lässt sowie geeignet ist, die Qualität dieser Dienstleistungscenter zu erfassen. 
Nicht berücksichtigt worden sind im vorliegenden Artikel schließlich mögliche NichtLinearitäten zwischen den einzelnen Konstrukten. So kann z. B. bezüglich des Centerbetriebs angenommen werden, dass er zu den Basisleistungen zählt. Die Funktionalität des Centers wird von den Centernutzern erwartet und stellt somit eine Mindestanforderung dar. Die Erfüllung der Kundenanforderungen an den Center ist für ein gutes Qualitätsurteil notwendig, reicht für sich genommen jedoch nicht aus. Daher ist zu vermuten, dass die Wirkungsbeziehung zwischen der Centerbetriebsqualität und Gesamtqualität des Dienstleistungscenters de-gressiv ist. Auch bei den Kontaktpunkten sind nicht-lineare Wirkungsbeziehungen denkbar. So ist es möglich, dass ein Kontaktpunkt den Nutzer begeistert, während die Qualität anderer Kontaktpunkte direkt proportional auf die Gesamtqualität einwirkt und wieder andere Kontaktpunkte nur Mindestanforderungen genügen. Dies gilt es künftig stärker zu berücksichtigen.

\section{Anmerkungen}

1 Ein Beleg hierfür ist u. a. die umfangreiche Literatur zu diesem Themengebiet. Zum Beispiel Zeithaml 1981, 2000; Parasuraman et al. 1985, 1988, 1991, 1993; Bitner 1990; Grönroos 1990; Cronin u. Taylor 1992, 1994; Haller 1993; Güthoff 1995; Zeithaml u. Bitner 1996, 2000; Olandt 1998; Dabholkar et al. 2000; Brady u. Cronin 2001; Riedmüller 2003; Zielke 2003; Bruhn 2006; Svensson 2006a; González et al. 2007; Ueltschy et al. 2007.

2 Diesem Dimensionierungsansatz wurde von Birkelbach im Rahmen einer Eignungsdiskussion besondere Bedeutung für Dienstleistungscenter zugesprochen. Im Folgenden wird auf diesen Ansatz fokussiert, zumal die Unterteilung in eine Potenzial-, Prozess- und Ergebnisqualität ohnehin andere Ansätze (wie z. B. den SERVQUAL-Ansatz von Parasuraman et al. 1988) implizit behandelt. Zur Auseinandersetzung mit den verschiedenen Ansätzen, vgl. Birkelbach 1993, S. 29 ff.).

3 Die Kontaktobjekte bzw. -subjekte stellen sich als die Bereitstellungsleistungen des Dienstleisters aus Nachfragersicht dar. Rosado 1990, S. 170 f.

4 Anzumerken ist an dieser Stelle, dass die Kontaktpunktzufriedenheit mit Hilfe von Qualitätsmerkmalen erfasst wird. Dies lässt die Verwendung des Begriffs „Kontaktpunktqualität“ eher zutreffend erscheinen.

5 Vgl. zum Einsatz der Beobachtung im Dienstleistungsmarketing, Grove u. Fisk 1992, S. 217 ff.

6 Als Experten wurden Mitarbeitende des Flughafenmanagements und der Fluggesellschaften angesehen, da diese aufgrund ihrer beruflichen Erfahrung über gute Kenntnisse der Kundenprozesse an einem Flughafen verfügen.

7 Damit wurde der Empfehlung von Botschen et al. gefolgt, die sich bei der Entwicklung eines Ablaufdiagramms aus Kundensicht für die Kombination aus persönlichen Interviews und teilnehmende Beobachtungen aussprechen. Botschen et al. 1996, S. 26.

8 Die C-OAR-SE-Prozedur ist nicht unumstritten. So wird zum Beispiel die von Rossiter empfohlene Single-Item-Messung infrage gestellt. Ebenso fehlen nach Auffassungen der Kritiker des Ansatzes Kriterien zur Auswahl formativer Messindikatoren. Weiterhin trifft Rossiter keine genauen Aussagen darüber, in welchem Bereich die Beurteiler Experten zu sein haben. Im Rahmen der vorliegenden Studie erfolgt dennoch eine Orientierung an C-OAR-SE, da der Ansatz dem Forscher im Vergleich zum Churchill-Prozess den Vorteil bietet, sich nicht auf ein rein kennziffernorientiertes Vorgehen bei der Indikatorenauswahl zu stützen, sondern das Augenmerk vielmehr auf die Gewährleistung einer möglichst hohen Inhaltsvalidität legt und 
somit gerade für die Entwicklung formativ gemessener Konstrukte, wie sie auch im vorliegenden Fall vorliegen, geeignet ist. Zur ausführlichen Kritik an C-OAR-SE, vgl. Diamantopoulos 2005, zum Churchill-Prozess der Konstruktoperationalisierung, vgl. Churchill 1979.

9 Vgl. zur Sequenziellen Ereignismethode Stauss u. Hentschel 1990, S. 244 ff.; Hentschel 1992, S. $169 \mathrm{ff}$. Eine auf dem Blueprint beruhende Strukturierung des Interviews bot sich hier an, da nach psychologischen Erkenntnissen um einzelne abgrenzbare Sequenzen (hier die identifizierten Kundenkontaktpunkte) Wissen abgespeichert wird, vgl. zur episodischen Informationsverarbeitung Lindsay u. Norman 1981, S. 303. Durch den Einsatz des Blueprints liefert die Sequenzielle Ereignismethode gestützte Erinnerungen (,aided recalls“).

10 Zur Unterscheidung formativ und reflektiv gemessener Konstrukte, vgl. Chin 1998a; Ringle 2004a; Fassott u. Eggert 2005; Riemenschneider 2006.

11 Die mangelnde Identifizierung des Bereichs technische Ausstattung deutet darauf hin, dass der Centernutzer diesen bei einem Flughafen nicht wahrnimmt.

12 Zum PLS-Algorithmus und zur Einführung in die PLS-Pfadmodellierung, vgl. Betzin u. Henseler 2005; Henseler 2005.

13 Zu den Vor- und Nachteilen varianz- bzw. kovarianzbasierter Verfahren, vgl. Chin et al. 2003.

14 Mit einem Wert von 0,769 liegt die Faktorladung von Item DLCQ_1 aber nur knapp unter dem geforderten Mindestwert von 0,8, so dass sich für dessen Beibehaltung entschieden wurde.

\section{Anhang}

Tab. 1: Messindikatoren der Centerbetriebsqualität

\begin{tabular}{lll}
\hline Indikatorbezeichnung & Indikatorformulierung & Bereich \\
\hline CBQ_1 & $\begin{array}{l}\text { Die Erreichbarkeit mit dem } \\
\text { PKW entspricht genau meinen } \\
\text { Vorstellungen. }\end{array}$ & Standort \\
& $\begin{array}{l}\text { Die Erreichbarkeit mit } \\
\text { öffentlichen Verkehrsmitteln ... }\end{array}$ & Standort \\
DBQ_2 & Die Beschilderung zum & Standort \\
FBQ_3 & Flughafen ... & \\
& Die Großzügigkeit des & Umfeld im Flughafengebäude \\
CBQ_4 & Flughafengebäudes ... & \\
Die Temperatur im & Umfeld im Flughafengebäude \\
CBQ_5 & Flughafengebäude ... & \\
Die Luftqualität im & Umfeld im Flughafengebäude \\
& Flughafengebäude ... & \\
Der Geräuschpegel im & Umfeld im Flughafengebäude \\
Flughafengebäude ... & \\
CBQ_8 & $\begin{array}{l}\text { Das Sicherheitsempfinden im } \\
\text { Flughafengebäude ... }\end{array}$ & Umfeld im Flughafengebäude \\
CBQ_9 & $\begin{array}{l}\text { Die Vielfalt der angeflogenen } \\
\text { Destinationen ... }\end{array}$ & Leistungsumfang \\
Die Häufigkeit der & Leistungsumfang \\
Flugerbindungen ... & \\
Die vertretenen & Leistungsumfang \\
& Fluggesellschaften ... & \\
\hline
\end{tabular}


Tab. 2: Messindikatoren der Kontaktpunktqualitäten

\begin{tabular}{|c|c|c|}
\hline $\begin{array}{l}\text { Konstrukt/ } \\
\text { Indikatorbezeichnung }\end{array}$ & Indikatorformulierung & Bereich \\
\hline \multicolumn{3}{|l|}{ Check-in-Qualität } \\
\hline Check_1 & $\begin{array}{l}\text { Die Freundlichkeit des Check-in-Perso- nals } \\
\text { entspricht genau meinen Vorstellungen. }\end{array}$ & Interaktion \\
\hline Check_2 & $\begin{array}{l}\text { Die Kompetenz des Check-in-Personals } \\
\text { entspricht genau meinen Vorstellungen. }\end{array}$ & Interaktion/Leistung \\
\hline Check_3 & $\begin{array}{l}\text { Die Einrichtung des Check-in-Bereichs entspricht } \\
\text { genau meinen Vorstellungen. }\end{array}$ & Leistung \\
\hline Check_4 & $\begin{array}{l}\text { Die Schnelligkeit des Check-ins entspricht genau } \\
\text { meinen Vorstellungen. }\end{array}$ & Leistung \\
\hline Check_5 & $\begin{array}{l}\text { Die stressfreie Abwicklung des Eincheckens } \\
\text { entspricht genau meinen Vorstellungen. }\end{array}$ & Leistung \\
\hline \multicolumn{3}{|l|}{ Passkontrollen-Qualität } \\
\hline Pass_1 & $\begin{array}{l}\text { Die Freundlichkeit des Personals an der } \\
\text { Passkontrolle entspricht genau meinen } \\
\text { Vorstellungen. }\end{array}$ & Interaktion \\
\hline Pass_2 & $\begin{array}{l}\text { Die Einrichtung im Passkontrollen-Bereich } \\
\text { entspricht genau meinen Vorstellungen. }\end{array}$ & Leistung \\
\hline Pass_3 & $\begin{array}{l}\text { Die Schnelligkeit der Passkontrolle entspricht } \\
\text { genau meinen Vorstellungen. }\end{array}$ & Leistung \\
\hline Pass_4 & $\begin{array}{l}\text { Die stressfreie Abwicklung der Passkontrolle } \\
\text { entspricht genau meinen Vorstellungen. }\end{array}$ & Leistung \\
\hline Pass_5 & $\begin{array}{l}\text { Die faire Behandlung durch den Beamten an der } \\
\text { Passkontrolle entspricht genau meinen } \\
\text { Vorstellungen. }\end{array}$ & Interaktion \\
\hline \multicolumn{3}{|l|}{ Security-Qualität } \\
\hline Sich_1 & $\begin{array}{l}\text { Die Freundlichkeit des Personals an der } \\
\text { Sicherheitskontrolle entspricht genau meinen } \\
\text { Vorstellungen. }\end{array}$ & Interaktion \\
\hline Sich_2 & $\begin{array}{l}\text { Die Kompetenz des Personals an der } \\
\text { Sicherheitskontrolle entspricht genau meinen } \\
\text { Vorstellungen. }\end{array}$ & Interaktion/Leistung \\
\hline Sich_3 & $\begin{array}{l}\text { Die Einrichtung des Security-Bereichs entspricht } \\
\text { genau meinen Vorstellungen. }\end{array}$ & Leistung \\
\hline Sich_4 & $\begin{array}{l}\text { Die Schnelligkeit der Sicherheitskontrolle } \\
\text { entspricht genau meinen Vorstellungen. }\end{array}$ & Leistung \\
\hline Sich_5 & $\begin{array}{l}\text { Die stressfreie Abwicklung der } \\
\text { Sicherheitskontrolle entspricht genau meinen } \\
\text { Vorstellungen. }\end{array}$ & Leistung \\
\hline \multicolumn{3}{|l|}{ Shopping-Qualität } \\
\hline Shop_1 & $\begin{array}{l}\text { Die Freundlichkeit des Verkaufspersonals } \\
\text { entspricht genau meinen Vorstellungen. }\end{array}$ & Interaktion \\
\hline Shop_2 & $\begin{array}{l}\text { Die Kompetenz des Verkaufspersonals entspricht } \\
\text { genau meinen Vorstellungen. }\end{array}$ & Interaktion/Leistung \\
\hline Shop_3 & $\begin{array}{l}\text { Die Vielfalt des Warenangebots entspricht genau } \\
\text { meinen Vorstellungen. }\end{array}$ & Leistung \\
\hline Shop_4 & $\begin{array}{l}\text { Die Annehmlichkeit des Besuchs im Geschäft } \\
\text { entspricht genau meinen Vorstellungen. }\end{array}$ & Leistung \\
\hline
\end{tabular}


Tab. 2: (Fortsetzung)

\begin{tabular}{|c|c|c|}
\hline $\begin{array}{l}\text { Konstrukt/ } \\
\text { Indikatorbezeichnung }\end{array}$ & Indikatorformulierung & Bereich \\
\hline \multicolumn{3}{|l|}{ Gastronomie-Qualität } \\
\hline Rest_1 & $\begin{array}{l}\text { Die Freundlichkeit der Mitarbeiter des } \\
\text { Restaurants/der Bar entspricht genau meinen } \\
\text { Vorstellungen. }\end{array}$ & Interaktion \\
\hline Rest_2 & $\begin{array}{l}\text { Die Kompetenz der Mitarbeiter des } \\
\text { Restaurants/der Bar entspricht genau meinen } \\
\text { Vorstellungen. }\end{array}$ & Interaktion/Leistung \\
\hline Rest_3 & $\begin{array}{l}\text { Die Auswahl an Speisen entspricht genau meinen } \\
\text { Vorstellungen. }\end{array}$ & Leistung \\
\hline Rest_4 & $\begin{array}{l}\text { Der Geschmack der Speisen entspricht genau } \\
\text { meinen Vorstellungen. }\end{array}$ & Leistung \\
\hline Rest_5 & $\begin{array}{l}\text { Die Entspannungsmöglichkeit im Restaurant/in } \\
\text { der Bar entspricht genau meinen Vorstellungen. }\end{array}$ & Leistung \\
\hline Rest_6 & $\begin{array}{l}\text { Die Annehmlichkeit des Restaurant-/Barbesuchs } \\
\text { entspricht genau meinen Vorstellungen. }\end{array}$ & Leistung \\
\hline \multicolumn{3}{|l|}{ Wartebereichs-Qualität } \\
\hline Wartb_1 & $\begin{array}{l}\text { Die Freundlichkeit des Personals entspricht genau } \\
\text { meinen Vorstellungen. }\end{array}$ & Interaktion \\
\hline Wartb_2 & $\begin{array}{l}\text { Die Kompetenz des Personals entspricht genau } \\
\text { meinen Vorstellungen. }\end{array}$ & Interaktion/Leistung \\
\hline Wartb_3 & $\begin{array}{l}\text { Der Komfort der Sitzmöglichkeiten entspricht } \\
\text { genau meinen Vorstellungen. }\end{array}$ & Leistung \\
\hline Wartb_4 & $\begin{array}{l}\text { Die Unterhaltungsmöglichkeiten im Wartebereich } \\
\text { entspricht genau meinen Vorstellungen. }\end{array}$ & Leistung \\
\hline Wartb_5 & $\begin{array}{l}\text { Das Zeitschriftenangebot entspricht genau } \\
\text { meinen Vorstellungen. }\end{array}$ & Leistung \\
\hline Wartb_6 & $\begin{array}{l}\text { Die Atmosphäre im Wartebereich entspricht } \\
\text { genau meinen Vorstellungen. }\end{array}$ & Leistung \\
\hline
\end{tabular}

Tab. 3: Messindikatoren der Dienstleistungscenterqualität

\begin{tabular}{lll}
\hline Indikatorbezeichnung & & Indikatorformulierung \\
\hline DLCQ_1 & Gesamtqualität & $\begin{array}{l}\text { Der Flughafen entspricht insgesamt } \\
\text { meinen Vorstellungen. }\end{array}$ \\
DLCQ_2 & Gesamtzufriedenheit & $\begin{array}{l}\text { Wie zufrieden sind Sie insgesamt } \\
\text { mit den Leistungen des Flughafens? }\end{array}$ \\
DLCQ_3 & Erfüllungsgrad der & $\begin{array}{l}\text { Wie sehr wurden Ihre Erwartungen } \\
\text { an den Flughafen erfüllt? }\end{array}$ \\
DLCQ_4 & Erwartungen & $\begin{array}{l}\text { Wie nahe kommt der Flughafen } \\
\text { dem Ideal? }\end{array}$ \\
\hline
\end{tabular}


Tab. 4: Ergebnisse der Messmodelle

\begin{tabular}{|c|c|c|c|}
\hline \multicolumn{4}{|c|}{ Formative Messmodelle } \\
\hline Konstrukt/Code & Item & Gewicht & T-Wert $(>1,98)$ \\
\hline \multicolumn{4}{|l|}{$\overline{\mathrm{CBQ}}$} \\
\hline CBQ_1 & Erreichbarkeit mit PKW & 0,120 & 2,617 \\
\hline CBQ_2 & $\begin{array}{l}\text { Erreichbarkeit mit öffentlichen } \\
\text { Verkehrsmitteln }\end{array}$ & $-0,050$ & 1,003 \\
\hline CBQ_3 & Beschilderung & 0,121 & 1,788 \\
\hline CBQ_4 & Gebäude & 0,292 & 5,810 \\
\hline CBQ_5 & Temperatur & 0,073 & 1,133 \\
\hline CBQ_6 & Luftqualität & 0,132 & 1,452 \\
\hline CBQ 7 & Geräuschpegel & 0,153 & 2,173 \\
\hline CBQ_8 & Sicherheitsempfinden & 0,273 & 4,166 \\
\hline CBQ_9 & Destinationen & 0,265 & 2,698 \\
\hline CBQ_10 & Häufigkeit Verbindungen & $-0,078$ & 0,674 \\
\hline CBQ_11 & Vertretene Fluggesellschaften & 0,204 & 2,007 \\
\hline \multicolumn{4}{|c|}{ Check-in-Qualität } \\
\hline Check_1 & Freundlichkeit des Personals & 0,295 & 3,890 \\
\hline Check_2 & Kompetenz des Personals & 0,145 & 2,216 \\
\hline Check_3 & Einrichtung Check-in-Bereich & 0,502 & 8,799 \\
\hline Check_4 & Schnelligkeit des Check-in & 0,155 & 2,145 \\
\hline Check_5 & Stressfreie Abwicklung & 0,271 & 3,319 \\
\hline \multicolumn{4}{|c|}{ Passkontrollen-Qualität } \\
\hline Pass_1 & Freundlichkeit des Personals & 0,508 & 3,945 \\
\hline Pass_2 & Einrichtung Passkontrollenbereich & 0,617 & 5,103 \\
\hline Pass_3 & Schnelligkeit der Passkontrolle & $-0,275$ & 1,626 \\
\hline Pass 4 & Stressfreie Abwicklung & 0,354 & 1,674 \\
\hline Pass_5 & Faire Behandlung & $-0,127$ & 0,711 \\
\hline \multicolumn{4}{|l|}{ Security-Qualität } \\
\hline Sich_1 & Freundlichkeit des Personals & 0,230 & 2,840 \\
\hline Sich_2 & Kompetenz des Personals & 0,321 & 4,739 \\
\hline Sich_3 & Einrichtung Security-Bereich & 0,520 & 6,980 \\
\hline Sich_4 & Schnelligkeit der Sicherheitskontrolle & 0,181 & 1,859 \\
\hline Sich_5 & Stressfreie Abwicklung & 0,034 & 0,318 \\
\hline \multicolumn{4}{|c|}{ Shopping-Qualität } \\
\hline Shop_1 & Freundlichkeit des Personals & $-0,014$ & 0,064 \\
\hline Shop_2 & Kompetenz des Personals & 0,324 & 1,572 \\
\hline Shop_3 & Vielfalt des Warenangebots & 0,101 & 0,578 \\
\hline Shop_4 & $\begin{array}{l}\text { Annehmlichkeit des Besuchs im } \\
\text { Geschäft }\end{array}$ & 0,648 & 3,381 \\
\hline
\end{tabular}


Tab. 4: (Fortsetzung)

\begin{tabular}{|c|c|c|c|}
\hline \multicolumn{4}{|c|}{ Formative Messmodelle } \\
\hline Konstrukt/Code & Item & Gewicht & T-Wert $(>1,98)$ \\
\hline \multicolumn{4}{|c|}{ Gastronomie-Qualität } \\
\hline Rest_1 & Freundlichkeit des Personals & $-0,151$ & 0,990 \\
\hline Rest_2 & Kompetenz des Personals & $-0,190$ & 1,239 \\
\hline Rest_3 & Auswahl an Speisen & 0,0190 & 0,117 \\
\hline Rest_4 & Geschmack der Speisen & 0,140 & 1,071 \\
\hline Rest_5 & Entspannungsmöglichkeit & 0,696 & 3,433 \\
\hline \multirow[t]{3}{*}{ Rest_6 } & Annehmlichkeit & 0,467 & 2,207 \\
\hline & Restaurants-/ & & \\
\hline & Barbesuchs & & \\
\hline \multicolumn{4}{|c|}{ Wartebereichs-Qualität } \\
\hline Wartb_1 & Freundlichkeit des Personals & 0,168 & 1,789 \\
\hline Wartb_2 & Kompetenz des Personals & 0,177 & 1,790 \\
\hline Wartb_3 & Komfort der Sitzmöglichkeiten & 0,268 & 4,024 \\
\hline Wartb_4 & Unterhaltungsmöglichkeiten & 0,052 & 0,643 \\
\hline Wartb_5 & Zeitschriftenangebot & 0,192 & 1,969 \\
\hline Wartb_6 & Atmosphäre & 0,417 & 4,416 \\
\hline \multicolumn{4}{|c|}{ Reflektives Messmodell } \\
\hline Konstrukt/Code & Item & Faktorladung & T-Wert $(>1,64)$ \\
\hline \multicolumn{4}{|l|}{ DLCQ } \\
\hline \multirow[t]{2}{*}{ DLCQ_1 } & Flughafen entspricht insgesamt & 0,769 & 26,354 \\
\hline & Vorstellungen & & \\
\hline DLCQ_2 & Gesamtzufriedenheit & 0,897 & 105,402 \\
\hline DLCQ_3 & Erwartungsabgleich & 0,870 & 49,441 \\
\hline DLCQ_4 & Ideal-Abgleich & 0,868 & 67,416 \\
\hline
\end{tabular}


Tab. 5: Varianzinflationswerte (VIF)

R-Quadrat reg.

Für VIF

\section{Ceck In}

Check_1

Check_2

Check_3

Check_4

Check_5

\section{Passkontrolle}

Pass_1

Pass_2

Pass_3

Pass_4

Pass_5

\section{Security}

Sich_1

Sich_2

Sich_3

Sich_4

Sich_5

Gastronomie

Rest_1

Rest_2

Rest_3

Rest_4

Rest_5

Rest_6

Shopping

Shop_1

Shop_2

Shop_3

Shop_4

\section{Wartebereich}

Wartb_1

Wartb_2

Wartb_3

Wartb_4

Wartb_5

Wartb_6

\section{Centerbetriebsqualität}

CBQ_1

CBQ_2

CBQ_3

CBQ_4

CBQ_5

CBQ_6

CBQ_7

CBQ_8

CBQ_9

CBQ_10

CBQ_11

$\begin{array}{ll}0,425 & 1,74 \\ 0,437 & 1,78 \\ 0,229 & 1,30 \\ 0,533 & 2,14 \\ 0,573 & 2,34\end{array}$

$0,418 \quad 1,72$

$0,381 \quad 1,62$

$0,665 \quad 2,99$

$0,717 \quad 3,53$

$0,587 \quad 2,42$

$0,446 \quad 1,81$

$0,409 \quad 1,69$

$0,381 \quad 1,62$

$0,609 \quad 2,56$

$0,649 \quad 2,85$

$0,592 \quad 2,45$

$0,577 \quad 2,36$

$0,53 \quad 2,13$

$0,504 \quad 2,02$

$0,733 \quad 3,75$

$0,763 \quad 4,22$

$0,497 \quad 1,99$

$0,552 \quad 2,23$

$0,477 \quad 1,91$

$0,56 \quad 2,27$

$0,603 \quad 2,52$

$0,587 \quad 2,42$

$0,416 \quad 1,71$

$0,57 \quad 2,33$

$0,542 \quad 2,18$

$0,602 \quad 2,51$

$0,238 \quad 1,31$

$0,117 \quad 1,13$

$0,297 \quad 1,42$

$0,39 \quad 1,64$

$0,588 \quad 2,43$

$0,637 \quad 2,75$

$0,546 \quad 2,20$

$0,439 \quad 1,78$

$0,648 \quad 2,84$

$0,722 \quad 3,60$

$0,63 \quad 2,70$ 


\section{Literatur}

Albers S, Hildebrandt L (2006) Methodische Probleme bei der Erfolgsfaktorenforschung - Messfehler, formative versus reflektive Indikatoren und die Wahl des Strukturgleichungs-Modells. Zfbf $58(2): 2-33$

Albrecht K (1988) At America's service: how corporations can revolutionize the way they treat their customers, business one irwin. Dow Jones-Irwin, Homewood

Baker J (1987) The role of the environment in marketing services: the consumer perspective. In: Czepiel JA, Congram CA, Shanahan J (Hrsg) The services challenge: integrating competitive advantage. American Marketing Association, Chicago, S 79-84

Baker J, Berry LL, Parasuraman A (1988) The impact of branch facility design. J Retail Bank $10(2): 33-42$

Bauer HH, Huber F, Majer T (2000) Zufriedenheitsdynamik und Kundenbindung bei Kundendienstleistungsprozessen im Handel - Ergebnisse einer empirischen Studie. In: Bruhn M, Stauss B (Hrsg) Kundenbeziehungen im Dienstleistungsbereich. Gabler, Wiesbaden, S 24-46

Bauer HH, Exler S, Reichardt T, Ringeisen P (2006) Der Einfluss der Dienstleistungsqualität auf die Einkaufsstättentreue - Ein empirischer Vergleich zwischen Deutschland und Spanien, Wissenschaftliches Arbeitspapier Nr. W 98, Institut für Marktorientierte Unternehmensführung, Universität Mannheim, Mannheim

Becker FD (1981) Workspace. Praeger, New York

Bell J, Gilbert D, Lockwood A (1997) Service quality in food retailing operations: a critical incident analysis. Int Rev Retail, Distribution Consum Res 7(4):405-423

Benkenstein M, Zielke K (2003) Messung von Qualitätsanforderungen in Dienstleistungsnetzwerken - dargestellt am Beispiel touristischer Dienstleistungen. In: Bruhn M, Stauss B (Hrsg) Dienstleistungsnetzwerke. Gabler, Wiesbaden, S 405-423

Betzin J, Henseler J (2005) Einführung in die Funktionsweise des PLS-Algorithmus. In: Bliemel F, Eggert A, Fassott G, Henseler J (Hrsg) Handbuch PLS-Pfadmodellierung. Schäffer-Poeschel, Stuttgart, S 49-70

Bigné E, Moliner MA, Sánchez J (2003) Perceived quality and satisfaction in multiservice organizations: the case of Spanish public services. J Serv Mark 17(4):420-442

Birkelbach R (1993) Qualitätsmanagement in Dienstleistungscentern. Lang, Frankfurt a.M.

Bitner MJ (1990) Evaluating service encounters: the effects of physical surroundings and employee responses. J Mark 54(2):69-82

Bitner MJ (1992) Servicescapes: the impact of physical surroundings on customers and employees. J Mark 56(2):57-71

Bost E (1987) Ladenatmosphäre und Konsumentenverhalten. Physica, Heidelberg

Botschen G, Bstieler L, Woodside AG (1996) Sequence-oriented problem identification within service encounters. J Euromarketing 5(2):19-52

Brady MK, Cronin JJ (2001) Some new thoughts on conceptualizing perceived service quality: a hierarchical approach. J Mark 65(3):34-49

Bruhn M (2006) Qualitätsmanagement für Dienstleistungen. Grundlagen, Konzepte, Methoden, 6 Aufl. Springer, Berlin

Chin WW (1998a) Issues and opinions on structural equation modeling. MIS Q 22(1):7-16

Chin WW (1998b) The partial least approach to structural equation modeling. In: Marcoulides GA (Hrsg) Modern methods for business research. Lawrence Erlbaum Associates, Mahwah, S 295-336

Chin WW (2003) PLSgraph. http://www.cba.uh.edu/plsgraph/

Chin WW, Newsted PR (1999) Structural equation modeling analysis with small samples using partial least squares. In: Hoyle RH (Hrsg) Strategies for small sample research. Sage, Thousand Oaks, S $307-347$

Chin WW, Marcolin BL, Newsted PR (2003) A partial least squares modeling approach for measuring interaction effects: results from a monte carlo simulation study and an electronic mail emotion/adoption study. Inf Syst Res 14(2):189-217 
Churchill GA (1979) A paradigm for developing better measures of marketing constructs. J Mark Res 16(1):64-73

Cronin JJ, Taylor SA (1992) Measuring service quality: a reexamination and extension. J Mark 56(3):55-68

Cronin JJ, Taylor SA (1994) SERVPERF versus SERVQUAL: reconciling performance-based and perceptions minus expectations measurement of service quality. J Mark 58(1):125-131

Dabholkar PA, Rentz JO, Thorpe DI (1996) A measure of service quality for retail stores: scale development and validation. J Acad Mark Sci 24(1):3-16

Dabholkar PA, Shepherd CD, Thorpe DI (2000) A comprehensive framework for service quality: an investigation of critical conceptual and measurement issues through a longitudinal study. $\mathrm{J}$ Retailing 76(2):139-173

Danaher PJ, Mattsson J (1994a) Customer satisfaction during the service delivery process. Eur J Mark 28(5):5-16

Danaher PJ, Mattsson J (1994b) Cumulative encounter satisfaction in the hotel conference process. Int J Serv Industry Manag 5(4):69-80

Diamantopoulos A (2005) The C-OAR-SE procedure for scale development in marketing: a comment. Int J Res Mark 22(1):1-9

Diamantopoulos A, Winklhofer HM (2001) Index construction with formative indicators: an alternative to scale development. J Mark Res 38(2):269-277

Donabedian A (1980) The definition of quality and approaches to its assessment. Explorations in quality, assessment and monitoring, vol 1. Health Administration Press, Ann Arbor

Fassott G, Eggert A (2005) Zur Verwendung formativer und reflektiver Indikatoren in Strukturgleichungsmodellen: Bestandsaufnahme und Anwendungsempfehlungen. In: Bliemel F, Eggert A, Fassott G, Henseler J. (Hrsg) Handbuch Pfadmodellierung. Methode, Anwendung, Praxisbeispiele. Schäffer-Poeschel, Stuttgart, S 31-47

Fornell C, Johnson MD, Anderson EW, Jaesung C, Bryant BE (1996) The American customer satisfaction index: nature, purpose, and findings. J Mark 60(4):7-18

Götz O, Liehr-Gobbers K (2004) Der Partial-Least-Squares (PLS)-Ansatz zur Analyse von Strukturgleichungsmodellen. In: Krafft M (Hrgs) Arbeitspapiere des Instituts für Marketing: Marketing Centrum Münster, Nr 2, S 1-32

Gonzàlez MEA, Comesaña LR, Brea JAF (2007) Assessing tourist behavioral intentions through perceived service quality and customer satisfaction. J Bus Res 60(2):153-160

Grönroos C (1983) Innovative marketing strategies and organization structure for service firms. In: Berry L, Shostack GL, Upah GD (Hrsg) Emerging perspectives on service marketing. American Marketing Association, Chicago, S 9-21

Grönroos C (1984) A service quality model and its marketing implications. Eur J Mark 18(4):36-44

Grönroos C (1990) Service management and marketing: managing the moments of truth in service competition. Lexington Books, Toronto

Grönroos C (2000) Service management and marketing. A customer relationship management approach, 2 Aufl. Wiley, Chichester

Grove SJ, Fisk RP (1992) Observational data collection methods for services marketing: an overview. J Acad Mark Sci 20(3):217-225

Güthoff J (1995) Qualität komplexer Dienstleistungen. Konzeption und empirische Analyse der Wahrnehmungsdimensionen. Gabler, Wiesbaden

Haller S (1993) Methoden zur Beurteilung von Dienstleistungsqualität. Überblick zum State of the Art. Zfbf 45(1):19-40

Haugtvedt CP, Wegener DT (1994) Message order effects in persuasion: an attitude strength perspective. J Consum Res 21(1):205-218

Henseler J (2005) Einführung in die PLS-Pfadmodellierung. Wirtschaftswissenschaftliches Studium 34(2):70-75

Hentschel B (1992) Dienstleistungsqualität aus Kundensicht: vom merkmals- zum ereignisorientierten Ansatz. Gabler, Wiesbaden 
Hentschel B (2000) Multiattributive Messung von Dienstleistungsqualität. In: Bruhn M, Stauss B (Hrsg) Dienstleistungsqualität. Konzepte, Methoden, Erfahrungen, 3 Aufl. Gabler, Wiesbaden, S 289-320

Herrmann A, Huber F, Kressmann F (2006) Varianz- und kovarianzbasierte Strukturgleichungsmodelle - Ein Leitfaden zu deren Spezifikation, Schätzung und Beurteilung. Zfbf 58(2):34-66

Holzherr A (2006) Dienstleistungsqualität - Relevanz unterschiedlicher Teilleistungen im Dienstleistungsprozess: Eine Messung der Qualitätswahrnehmung mit einem teilleistungsbezogenen Ansatz, dissertation.de, Berlin

Homburg Ch, Fürst A (2008) Überblick über die Messung von Kundenzufriedenheit und Kundenbindung. In: Bruhn M, Homburg Ch (Hrsg) Handbuch Kundenbindungsmanagement. Grundlagen, Konzepte, Erfahrungen, 6 Aufl. Gabler, Wiesbaden, S 607-642

Jayawardhena C, Souchon A, Glanville K (2003) Service encounter quality in a business-to-business context: measure development and consequences. Paper presented at the Anzmac Conference

Jayawardhena C, Souchon AL, Farrell AM, Glanville K (2007) Outcomes of service encounter quality in a business-to-business context. Industrial Marketing Management 36(5):575-588

Lindsay PH, Norman DA (1981) Einführung in die Psychologie, 2 Aufl. Springer, Berlin

Meffert H, Birkelbach R (2000) Qualitätsmanagement in Dienstleistungszentren - Konzeptionelle Grundlagen und typenspezifische Ausgestaltung. In: Bruhn M, Stauss B (Hrsg) Dienstleistungsqualität. Konzepte, Methoden, Erfahrungen, 3 Aufl. Gabler, Wiesbaden, S 169-199

Olandt H (1998) Dienstleistungsqualität in Krankenhäusern. Operationalisierung und Messung der Patientenwahrnehmung. Gabler, Wiesbaden

Oliver RL (1977) The effects of expectations and disconfirmations on post-exposure product evaluations. J Appl Psychol 62(2):246-250

Oliver RL (1996) Satisfaction: a behavioral perspective on the consumer. McGraw-Hill Companies, New York

Owlia MS, Aspinwall EM (1998) A framework for measuring quality in engineering education. Total Qual Manag 9(6):501-518

Parasuraman A, Zeithaml VA, Berry LL (1985) A conceptual model of service quality and its implications for future research. J Mark 49(2):41-50

Parasuraman A, Zeithaml VA, Berry LL (1988) SERVQUAL: a multiple-item scale for measuring consumer perceptions of service quality. J Retail 64(1):12-40

Parasuraman A, Zeithaml VA, Berry LL (1991) Refinement and reassessment of the SERVQUAL scale. J Retail 67(4):420-450

Parasuraman A, Zeithaml VA, Berry LL (1993) Research note: more on improving service quality measurement. J Retail 69(1):141-147

Raajpoot N (2004) Reconceptualizing service encounter quality in non-western context. J Serv Res $7(2): 181-201$

Riedmüller F (2003) Dienstleistungsqualität bei professionellen Sportveranstaltungen. Entwicklung und Überprüfung eines Erklärungsmodells. Lang, Frankfurt a.M.

Riemenschneider M (2006) Der Wert von Produktvielfalt: Wirkung großer Sortimente auf das Verhalten von Konsumenten. Gabler, Wiesbaden

Ringle CM (2004a) Gütemaße für den Partial Least Squares-Ansatz zur Bestimmung von Kausalmodellen, Arbeitspapier Nr. 16, Institut für Industriebetriebslehre und Organisation. Universität Hamburg, Hamburg

Ringle CM (2004b) Messung von Kausalmodellen. Ein Methodenvergleich, Arbeitspapier Nr. 14, Institut für Industriebetriebslehre und Organisation. Universität Hamburg, Hamburg

Rosado M (1990) Kundendienststrategien im Automobilsektor. Duncker \& Humblot, Berlin

Rossiter JR (2002) The C-OAR-SE procedure for scale development in marketing. Int J Res Mark 19(4):305-335

Shostack GL (2001) How to design a service. Eur J Mark 16(1):49-63

Siu NYM, Cheung JT-H (2001) A measure of retail service quality. Mark Intell Plan 19(2):88-96 
Spintig S (2003) Beziehungsmanagement in Dienstleistungsnetzwerken, Arbeitspapier Nr. 104 des Betriebswirtschaftlichen Instituts, Universität Erlangen-Nürnberg, Nürnberg

Stadler M, Seeger F, Raeithel A (1977) Psychologie der Wahrnehmung. Grundfragen der Psychologie, 2 Aufl. Juventa, München

Stauss B, Hentschel B (1990) Verfahren der Problementdeckung und -analyse im Qualitätsmanagement von Dienstleistungen. Jahrbuch der Absatz- und Verbrauchsforschung 36:232-259

Sureshchandar GS, Rajendran C, Anatharaman RN (2003) Customer perceptions of service quality in the banking sector of a developing economy: a critical analysis. Int J Bank Mark 21(5):233-242

Svensson G (2004) A customized construct of sequential service quality in service encounter chains: time, context, and performance thresholds. Manag Serv Qual 14(6):468-475

Svensson G (2006a) New aspects of research into service encounters and service quality. Int J Serv Industry Manage 17(3/4):245-257

Svensson G (2006b) Sequential service quality in service encounter chains: case studies. J Serv Mark 20(1):51-58

Ueltschy LC, Laroche M, Eggert A, Bindi U (2007) Service quality and satisfaction: an international comparison of professional services perceptions. J Serv Mark 21(6):410-423

Wöhler K (2001) Tourismusmarketing. In: Tscheulin DK, Helmig B (Hrsg) Branchenspezifisches Marketing. Grundlagen, Besonderheiten, Gemeinsamkeiten. Gabler, Wiesbaden, S 188-200

Woratschek H, Roth S, Pastowski S (2003) Kooperation und Konkurrenz in Dienstleistungsnetzwerken. Eine Analyse am Beispiel des Destinationsmanagements. In: Bruhn M, Stauss B (Hrsg) Dienstleistungsnetzwerke. Gabler, Wiesbaden, S 253-284

Zeithaml VA (1981) How customer evaluation processes differ between goods and services. In: Donnelly JH, George WR (Hrsg) Marketing of services. American Marketing Association, Chicago, S 186-190

Zeithaml VA (2000) Service quality, profitability and economic worth of customers: what we know and what we need to learn. J Acad Mark Sci 28(1):67-86

Zeithaml VA, Bitner MJ (1996) Services marketing. McGraw-Hill, New York

Zeithaml VA, Bitner MJ (2000) Services marketing, 2 Aufl. McGraw-Hill, New York

Zielke K (2003) Qualität komplexer Dienstleistungsbündel. Ein mehrstufiger Ansatz zur Operationalisierung der Qualitätswahrnehmung am Beispiel touristischer Dienstleistungen. Gabler, Wiesbaden

\section{Measurement of quality in services centers - an investigation in the airport sector}

Abstract: The study shows that customers' perception of overall quality in services centers is driven by two components: the service encounter quality and the quality of the servicecape. Thus, customers' overall evaluation of service quality in a services center is a function of $\mathrm{n}$ service encounter qualities $(\mathrm{k}=1 \ldots \mathrm{n})$ and the quality of the servicecape. Furthermore, it is of great importance for the quality management of a services center that there could be (1) direct effects between the quality dimensions and the overall service quality as well as (2) direct effects between the quality dimensions themselves. The above mentioned study had been carried out exemplarily at the EuroAirport Basel-MulhouseFreiburg. The survey results suggest that servicecape quality has a significant effect both on the overall service quality and on the service encounter qualities. Moreover, the study shows that key quality indicators of the service center apply to the perception of security as well as to the service environment (for instance, arrangement of check-in/security desk, comfort of seats, ambience of waiting area).

Keywords: Quality in services centers · Service encounter quality · Quality of the servicecape 